Geometry $\&$ Topology

Volume 8 (2004) 831-876

Published: 2 June 2004

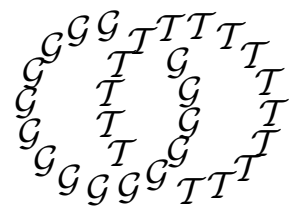

\title{
Heegaard splittings of graph manifolds
}

\author{
JenNifer SCHultens \\ Department of Mathematics \\ 1 Shields Avenue \\ University of California \\ Davis, CA 95616, USA \\ Email: jcs@math.ucdavis.edu
}

\begin{abstract}
Let $M$ be a totally orientable graph manifold with characteristic submanifold $\mathcal{T}$ and let $M=V \cup_{S} W$ be a Heegaard splitting. We prove that $S$ is standard. In particular, $S$ is the amalgamation of strongly irreducible Heegaard splittings. The splitting surfaces $S_{i}$ of these strongly irreducible Heegaard splittings have the property that for each vertex manifold $N$ of $M, S_{i} \cap N$ is either horizontal, pseudohorizontal, vertical or pseudovertical.
\end{abstract}

\section{AMS Classification numbers Primary: 57N10}

Secondary: 57N25

Keywords: Graph manifolds, Heegaard splitting, horizontal, vertical

Proposed: Cameron Gordon

Seconded: Joan Birman, Wolfgang Metzler
Received: 30 June 2003

Revised: 1 June 2004 


\section{Introduction}

The subject of this investigation is the structure of Heegaard splittings of graph manifolds. This investigation continues the work begun in [20, [21, [13] and 22. Since the publication of those papers, new techniques have been added to the repertoire of those interested in describing the structure of Heegaard splittings. These include the idea of untelescoping a weakly reducible Heegaard splitting into a generalized strongly irreducible Heegaard splitting due to $\mathrm{M}$ Scharlemann and A Thompson. They also include the Rubinstein-Scharlemann graphic, as employed by D Cooper and M Scharlemann in [6]. These insights have not left the investigation here unaffected. We hope that their role here is a tribute to proper affinag 1 . (The structural theorem given here has been promised for rather a long time.) A similar theorem was announced by $\mathrm{JH}$ Rubinstein.

The main theorems are the following, for defintions see Sections 2, 3, 4 and 5:

Theorem 1.1 Let $M$ be a totally orientable generalized graph manifold. If $M=V \cup_{S} W$ is a strongly irreducible Heegaard splitting, then $S$ is standard.

More specifically, $S$ can be isotoped so that for each vertex manifold $M_{v}$ of $M$, $S \cap M_{v}$ is either horizontal, pseudohorizontal, vertical or pseudovertical and such that for each edge manifold $M_{e}, S \cap M_{e}$ is characterized by one of the following:

(1) $S \cap M_{e}$ is a collection of incompressible annuli (including spanning annuli and possibly boundary parallel annuli) or is obtained from such a collection by ambient 1-surgery along an arc which is isotopic into $\partial M_{e}$.

(2) $\quad M_{e}$ is homeomorphic to (torus) $\times I$ and there is a pair of simple closed curves $c, c^{\prime} \subset($ torus $)$ such that $c \cap c^{\prime}$ consists of a single point $p \in($ torus $)$ and either $V \cap(($ torus $) \times I)$ or $W \cap(($ torus $) \times I)$ is a collar of $(c \times\{0\}) \cup(p \times I) \cup$ $\left(c^{\prime} \times\{1\}\right)$.

In the general case we can say the following:

Theorem 1.2 Let $M$ be a totally orientable graph manifold. Let $M=V \cup_{S} W$ be an irreducible Heegaard splitting. Let $M=\left(V_{1} \cup_{S_{1}} W_{1}\right) \cup_{F_{1}}\left(V_{2} \cup_{S_{2}} W_{2}\right) \cup_{F_{2}}$ $\cdots \cup_{F_{m-1}}\left(V_{m} \cup_{S_{m}} W_{m}\right)$ be a weak reduction of $M=V \cup_{S} W$. Set $M_{i}=V_{i} \cup W_{i}$. Then $M_{i}$ is a totally orientable generalized graph manifold and $M_{i}=V_{i} \cup_{S_{i}} W_{i}$

\footnotetext{
${ }^{1}$ This is a French noun describing the maturing process of a cheese.
} 
is a strongly irreducible Heegaard splitting. In particular, $M_{i}=V_{i} \cup_{S_{i}} W_{i}$ is standard.

Here $\chi(S)=\sum_{i}\left(\chi\left(S_{i}\right)-\chi\left(F_{i}\right)\right)$.

Theorem 1.3 Let $M$ be a totally orientable graph manifold. If $M=V \cup_{S} W$ is an irreducible Heegaard splitting, then it is the amalgamation of standard Heegaard splittings of generalized graph submanifolds of $M$.

The graph manifolds considered here are totally orientable, that is, they are orientable 3-manifolds and for each vertex manifold the underlying surface of the orbit space is orientable. It follows from [10, VI.34] that an incompressible surface can be isotoped to be either horizontal or vertical in each vertex manifold of a totally orientable graph manifold. In conjunction with the notion of untelescoping a weakly reducible Heegaard splitting into a strongly irreducible generalized Heegaard splitting, this observation reduces the investigation at hand to the investigation of strongly irreducible Heegaard splittings of generalized graph manifolds (for definitions, see below).

In the investigation of strongly irreducible Heegaard splittings of generalized graph manifolds, the nice properties of strongly irreducible Heegaard splittings often reduce this investigation to a study of the behaviour of the Heegaard splittings near the characteristic submanifolds. In this context, a theorem of D Cooper and M Scharlemann completes the description of this behaviour, see Proposition 7.15 and Proposition [7.23. This theorem may be found in 6 . Theorem 4.2].

The theorem here is purely structural in the sense that it describes the various ways in which a Heegaard splitting can be constructed. Specifically, there are finitely many possible constructions. Thus a totally orientable graph manifold possesses only finitely many Heegaard splittings up to isotopy (and hence also up to homeomorphism). It would be possible to extract a formula for the genera of these Heegaard splittings. However, this formula would be long, cumbersome and not very enlightening. But note that, in particular, the program here enables a computation of Heegaard genus, ie, the smallest possible genus of a Heegaard splitting, for totally orientable graph manifolds. To compute this genus, one need merely consider the finitely many possible constructions, compute the corresponding Euler characteristics, and find the extremal value. This line of thought is pursued in [24], where the genus of a certain class of totally orientable graph manifolds is compared to the rank, ie, the least number of generators, of the fundamental group of these manifolds. 
The theorem leaves open the question of classification. There will be some, though probably not too many, cases in which the various constructions are isotopic. More interestingly, there may be larger scale isotopies. Ie, there may be two Heegaard splittings of a graph manifold that are isotopic but not via an isotopy fixing their intersection with the decomposing tori. Clearly, this leaves much room for further investigation.

The global strategy is as follows: By Theorem 3.10, a Heegaard splitting is the amalgamation of the strongly irreducible Heegaard splittings arising in any of its weak reductions. Thus, one begins with a Heegaard splitting of a graph manifold. One then considers a weak reduction of this Heegaard splitting. Cutting along the incompressible surfaces in the weak reduction yields generalized graph manifolds with strongly irreducible Heegaard splittings. One analyzes the possible strongly irreducible Heegaard splittings of generalized graph manifolds. Finally, one considers all possibilities arising in the amalgamation of strongly irreducible Heegaard splittings of generalized graph manifolds.

I wish to thank the many colleagues who have reminded me that a complete report on this investigation is past due. Among these are Ian Agol, Hugh Howards, Yoav Moriah, Marty Scharlemann, Yo'av Rieck, Eric Sedgwick and Richard Weidmann. I also wish to thank the MPIM-Bonn where part of this work was done. This work was supported in part by the grant NSF-DMS 0203680 .

\section{Totally orientable graph manifolds}

For standard definitions pertaining to knot theory see for instance [4, 11] or [15]. For 3-manifolds see [9] or [10]. Note that the terminology for graph manifolds has not been standardized.

Definition 2.1 A Seifert manifold is a compact 3-manifold that admits a foliation by circles.

For a more concrete definition, see for instance [10]. The fact that the simple definition here is in fact equivalent to more concrete definitions follows from [7].

Definition 2.2 The circles in the foliation of a Seifert fibered space $M$ are called fibers. The natural projection that sends each fiber to a point is denoted by $p: M \rightarrow Q$. The quotient space $Q$ is called the base orbifold of $M$. A fiber $f$ is called an exceptional fiber if nearby fibers wind around $f$ more than once. 
Otherwise, $f$ is called a regular fiber. The image under $p$ of a regular fiber is called a regular point and the image under $p$ of an exceptional fiber is called an exceptional point.

The base orbifold is in fact a surface. This follows from standard facts about foliations in conjunction with [7]. It also follows that there will be only finitely many exceptional fibers.

Definition 2.3 For $Y$ a submanifold of $X$, we denote an open regular neighborhood of $Y$ in $X$ by $\eta(Y, X)$, or simply by $\eta(Y)$, if there is no ambiguity concerning the ambient manifold. Similarly, we denote a closed regular neighborhood by $N(Y, X)$, or simply by $N(Y)$, if there is no ambiguity concerning the ambient manifold.

Definition 2.4 A surface $S$ in a Seifert fibered space $M$ is vertical if it consists of fibers. It is horizontal if it intersects all fibers transversely. It is pseudohorizontal if there is a fiber $f \subset M$ such that $S \cap(M \backslash \eta(f))$ is horizontal and $S \cap N(f)$ is a collar of $f$.

It follows that a horizontal surface in a Seifert fibered space $M$ orbifold covers the base orbifold of $M$.

Definition 2.5 A Seifert fibered space is totally orientable if it is orientable as a $3-$-manifold and has an orientable base orbifold.

We are now ready to define graph manifolds.

Definition 2.6 A graph manifold is a 3-manifold $M$ modelled on a finite graph $\Gamma$ as follows:

(1) Each vertex $v$ of $\Gamma$ corresponds to a Seifert fibered space, denoted by $M_{v}$ and called a vertex manifold;

(2) Each edge $e$ of $\Gamma$ corresponds to a 3-manifold homeomorphic to (torus) $\times$ $S^{1}$, denoted by $M_{e}$ and called an edge manifold;

(3) If an edge $e$ is incident to a vertex $v$, then this incidence is realized by an identification of a boundary component of $M_{e}$ with a boundary component of $M_{v}$ via a homeomorphism.

A graph manifold is totally orientable if each vertex manifold is totally orientable. 
The union of edge manifolds in $M$ is also called the characteristic submanifold of $M$. It is denoted by $\mathcal{E}$. The image of a boundary component of the characteristic submanifold of $M$ is a torus called a decomposing torus. It is denoted by $\mathcal{T}$.

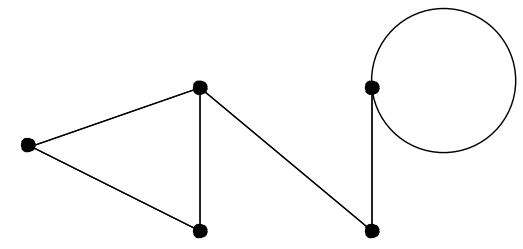

Figure 1: A model graph for a graph manifold

A decomposing torus is, of course, also the image of a boundary component of a vertex manifold. But the converse is not always true.

Remark 2.7 We have placed no restrictions on the homeomorphism that identifies a boundary component of an edge manifold with a boundary component of a vertex manifold. Thus according to this definition, there will be Seifert fibered spaces that admit a description as a graph manifold with non empty characteristic submanifold. From the point of view of the investigation here, this is often a useful way to think of such a Seifert fibered space. See [26].

Definition 2.8 A boundary component of a vertex manifold $M_{v}$ of a graph manifold $M$ that is also a boundary component of $M$ is called an exterior boundary component of $M_{v}$. We denote the union of exterior boundary components of $M_{v}$ by $\partial_{E} M_{v}$.

\section{Untelescoping and amalgamation}

We here give the basic definitions concerning Heegaard splittings, strongly irreducible Heegaard splittings, untelescopings and amalgamations. Theorem 3.10 below is crucial to the global strategy employed in our investigation.

Definition 3.1 A compression body is a 3-manifold $W$ obtained from a connected closed orientable surface $S$ by attaching 2-handles to $S \times\{0\} \subset S \times I$ and capping off any resulting 2 -sphere boundary components. We denote $S \times\{1\}$ by $\partial_{+} W$ and $\partial W \backslash \partial_{+} W$ by $\partial_{-} W$. Dually, a compression body is a connected 
orientable 3 -manifold obtained from a (not necessarily connected) closed orientable surface $\partial_{-} W \times I$ or a 3 -ball by attaching 1 -handles.

In the case where $\partial_{-} W=\emptyset$ (ie, in the case where a 3 -ball was used in the dual construction of $W$ ), we also call $W$ a handlebody. If $W=\partial_{-} W \times I$, we say that $W$ is a trivial compression body.

Definition 3.2 A spine of a compression body $W$ is a 1-complex $X$ such that $W$ collapses to $\partial_{-} W \cup X$.

Definition 3.3 A set of defining disks for a compression body $W$ is a set of disks $\left\{D_{1}, \ldots, D_{n}\right\}$ properly imbedded in $W$ with $\partial D_{i} \subset \partial_{+} W$ for $i=1$, $\ldots, n$ such that the result of cutting $W$ along $D_{1} \cup \cdots \cup D_{n}$ is homeomorphic to $\partial_{-} W \times I$ or to a 3 -ball in the case that $W$ is a handlebody.

Definition 3.4 A Heegaard splitting of a $3-$ manifold $M$ is a decomposition $M=V \cup_{S} W$ in which $V, W$ are compression bodies, $V \cap W=\partial_{+} V=\partial_{+} W=$ $S$ and $M=V \cup W$. We call $S$ the splitting surface or Heegaard surface.

The notion of strong irreducibility of a Heegaard splitting was introduced by A. Casson and C. McA. Gordon in [5] and has proven extremely useful.

Definition 3.5 A Heegaard splitting $M=V \cup_{S} W$ is strongly irreducible if for any pair of essential disks $D \subset V$ and $E \subset W, \partial D \cap \partial E \neq \emptyset$.

Recall also the following related definitions:

Definition 3.6 A Heegaard splitting $M=V \cup_{S} W$ is reducible if there exists a pair of essential disks $D \subset V$ and $E \subset W$ such that $\partial D=\partial E$. If $M=V \cup_{S} W$ is not reducible, then it is irreducible.

A Heegaard splitting $M=V \cup_{S} W$ is stabilized if there exists a pair of essential disks $D \subset V$ and $E \subset W$ such that $|\partial D \cap \partial E|=1$.

Though all compact 3-manifolds admit Heegaard splittings, many do not admit strongly irreducible Heegaard splitting. This fact prompted M. Scharlemann and A. Thompson to introduce the following notion of generalized Heegaard splittings. 
Definition 3.7 A generalized Heegaard splitting of a compact orientable 3manifold $M$ is a decomposition $M=\left(V_{1} \cup_{S_{1}} W_{1}\right) \cup_{F_{1}}\left(V_{2} \cup_{S_{2}} W_{2}\right) \cup_{F_{2}} \cdots \cup_{F_{m-1}}$ $\left(V_{m} \cup_{S_{m}} W_{m}\right)$ such that each of the $V_{i}$ and $W_{i}$ is a union of compression bodies with $\partial_{+} V_{i}=S_{i}=\partial_{+} W_{i}$ and $\partial_{-} W_{i}=F_{i}=\partial_{-} V_{i+1}$.

We say that a generalized Heegaard splitting is strongly irreducible if each Heegaard splitting of a component of $M_{i}=V_{i} \cup_{S_{i}} W_{i}$ is strongly irreducible and each $F_{i}$ is incompressible in $M$. We will denote $\cup_{i} F_{i}$ by $\mathcal{F}$ and $\cup_{i} S_{i}$ by $\mathcal{S}$. The surfaces in $\mathcal{F}$ are called the thin levels and the surfaces in $\mathcal{S}$ the thick levels.

Let $M=V \cup_{S} W$ be an irreducible Heegaard splitting. We may think of $M$ as being obtained from $\partial_{-} V \times I$ by attaching all 1-handles in $V$ (dual definition of compression body) followed by all 2 -handles in $W$ (standard definition of compression body), followed, perhaps, by 3-handles. An untelescoping of $M=$ $V \cup_{S} W$ is a rearrangement of the order in which the 1 -handles of $V$ and the 2-handles of $W$ are attached yielding a generalized Heegaard splitting. A weak reduction of $M=V \cup_{S} W$ is a strongly irreducible untelescoping of $M=V \cup_{S} W$.

Note that a weak reduction of a strongly irreducible Heegaard splitting would just be the strongly irreducible Heegaard splitting itself. The Main Theorem in 18 implies the following:

Theorem 3.8 Let $M$ be an irreducible 3-manifold. Any Heegaard splitting $M=V \cup_{S} W$ has a weak reduction.

Definition 3.9 Let $N, L$ be 3 -manifolds with $R$ a closed subsurface of $\partial N$, and $S$ a closed subsurface of $\partial L$, such that $R$ is homeomorphic to $S$ via a homeomorphism $h$. Further, let $\left(U_{1}, U_{2}\right),\left(V_{1}, V_{2}\right)$ be Heegaard splittings of $N, L$ such that $N(R) \subset U_{1}, N(S) \subset V_{1}$. Then, for some $R^{\prime} \subset \partial N \backslash R$ and $S^{\prime} \subset \partial L \backslash S, U_{1}=N\left(R \cup R^{\prime}\right) \cup(1$-handles $)$ and $V_{1}=N\left(S \cup S^{\prime}\right) \cup(1$-handles $)$. Here $N(R)$ is homeomorphic to $R \times I$ via a homeomorphism $f$ and $N(S)$ is homeomorphic to $S \times I$ via a homeomorphism $g$. Let $\sim$ be the equivalence relation on $N \cup L$ generated by

(1) $x \sim y$ if $x, y \epsilon \eta(R)$ and $p_{1} \cdot f(x)=p_{1} \cdot f(y)$,

(2) $x \sim y$ if $x, y \epsilon \eta(S)$ and $p_{1} \cdot g(x)=p_{1} \cdot g(y)$,

(3) $x \sim y$ if $x \epsilon R, y \epsilon S$ and $h(x)=y$, 
where $p_{1}$ is projection onto the first coordinate. Perform isotopies so that for $D$ an attaching disk for a 1 -handle in $U_{1}, D^{\prime}$ an attaching disk for a $1-$ handle in $V_{1},[D] \cap\left[D^{\prime}\right]=\emptyset$. Set $M=(N \cup L) / \sim, W_{1}=\left(U_{1} \cup V_{2}\right) / \sim$, and $W_{2}=\left(U_{2} \cup V_{1}\right) / \sim$. In particular, $(N(R) \cup N(S) / \sim) \cong R, S$. Then $W_{1}=V_{2} \cup N\left(R^{\prime}\right) \cup(1-$-handles $)$, where the 1 -handles are attached to $\partial_{+} V_{2}$ and connect $\partial N\left(R^{\prime}\right)$ to $\partial_{+} V_{2}$, and hence $W_{1}$ is a compression body. Analogously, $W_{2}$ is a compression body. So $\left(W_{1}, W_{2}\right)$ is a Heegaard splitting of $M$. The splitting $\left(W_{1}, W_{2}\right)$ is called the amalgamation of $\left(U_{1}, U_{2}\right)$ and $\left(V_{1}, V_{2}\right)$ along $R$, S via $h$.

Theorem 3.8 together with [20, Proposition 2.8] implies the following:

Theorem 3.10 Suppose $M=V \cup_{S} W$ is an irreducible Heegaard splitting and $M=\left(V_{1} \cup_{S_{1}} W_{1}\right) \cup_{F_{1}}\left(V_{2} \cup_{S_{2}} W_{2}\right) \cup_{F_{2}} \cdots \cup_{F_{m-1}}\left(V_{m} \cup_{S_{m}} W_{m}\right)$ a weak reduction of $M=V \cup_{S} W$. Then the amalgamation of $M=\left(V_{1} \cup_{S_{1}} W_{1}\right) \cup_{F_{1}}$ $\left(V_{2} \cup_{S_{2}} W_{2}\right) \cup_{F_{2}} \cdots \cup_{F_{m-1}}\left(V_{m} \cup_{S_{m}} W_{m}\right)$ along $F_{1} \cup \cdots \cup F_{m-1}$ is $M=V \cup_{S} W$.

One of the nice properties of strongly irreducible Heegaard splittings is apparent in the following lemma which is a deep fact and is proven, for instance, in [23, Lemma 6].

Lemma 3.11 Suppose $M=V \cup_{S} W$ is a strongly irreducible Heegaard splitting and $P \subset M$ an essential incompressible surface. Then $S$ can be isotoped so that $S \cap P$ consists only of curves essential in both $S$ and $P$.

\section{Incompressible surfaces and generalized graph manifolds}

In the arguments that follow, we employ the ideas of untelescoping and amalgamation. In this section, we describe the incompressible surfaces that arise in a weak reduction of a Heegaard splitting. We then describe the 3-manifolds that result from cutting a graph manifold along such incompressible surfaces. We will call these $3-$ manifolds generalized graph manifolds. Later, we will consider the strongly irreducible Heegaard splittings on these generalized graph manifolds.

Remark 4.1 An edge manifold of a totally orientable graph manifold $M$ is homeomorphic to $($ torus $) \times I$. There are infinitely many distinct foliations of 
(torus) $\times I$ as an annulus bundle over the circle. The incompressible surfaces in $($ torus $) \times I$ are tori isotopic to $($ torus $) \times\{$ point $\}$, annuli isotopic to the annular fibers in the foliations of (torus) $\times I$ as an annulus bundle over the circle and annuli parallel into $\partial(($ torus $) \times I)$.

Lemma 4.2 Let $F$ be an incompressible surface in a totally orientable graph manifold $M$. Then $F$ may be isotoped so that in each edge manifold it consists of incompressible tori and essential annuli and in each vertex manifold it is either horizontal or vertical.

Proof Let $\mathcal{T}$ be the collection of decomposing tori for $M$. Since $F$ and $\mathcal{T}$ are incompressible, $F$ may be isotoped so that $F \cap \mathcal{T}$ consists only of curves essential in both $F$ and $\mathcal{T}$. We may assume that this has been done in such a way that the number of components in $F \cap \mathcal{T}$ is minimal. Let $N$ be a component of $M \backslash \mathcal{T}$, then $F \cap N$ is incompressible. Furthermore, no component of $F \cap N$ is an annulus parallel into $\mathcal{T}$.

Suppose $F \cap N$ is boundary compressible in $N$. Let $\hat{D}$ be a boundary compressing disk for $F \cap N$. Then $\partial \hat{D}=a \cup b$, with $a \subset \partial N$ and $b \subset F$. Since $F \cap \mathcal{T}$ consists only of curves essential in both $F$ and $\mathcal{T}$, the component $A$ of $\partial N \backslash(F \cap \partial N)$ that contains $a$ is an annulus. Let $B(\hat{D})$ be a bicollar of $\hat{D}$. Then $\partial B(\hat{D})$ has two components, $\hat{D}_{0}, \hat{D}_{1}$. Consider the disk $D=\left(A \backslash(A \cap B(\hat{D})) \cup \hat{D}_{0} \cup \hat{D}_{1}\right.$. Since $F$ is incompressible, $D$ must be parallel to a disk in $F$, but this implies that the number of components of $F \cap \mathcal{T}$ is not minimal, a contradiction. Thus, $F \cap N$ is boundary incompressible in $N$.

If $N$ is an edge manifold, then $F \cap N$ is as required by Remark 4.1 If $N$ is a vertex manifold, then [10, VI.34] allows three possibilities for $F \cap N$ : (1) $F \cap N$ is vertical; (2) $F \cap N$ is horizontal; or (3) $F \cap N$ is the boundary of a twisted $I$-bundle over a horizontal surface $\hat{F}^{N}$ in $N$. For a boundary incompressible surface in $N$ this latter possibility would imply that there is a nonorientable horizontal surface $\hat{F}^{N}$ in $C$. In particular, $\hat{F}^{N}$ would be a cover of the base orbifold. But this is impossible. Hence $F \cap N$ is either horizontal or vertical. Hence $F \cap N$ is as required.

The following definition describes the 3-manifolds that result when a totally orientable graph manifold is cut along incompressible surfaces.

Definition 4.3 A generalized graph manifold is a 3-manifold $M$ modelled on a finite graph $\Gamma$ as follows: 
(1) Each vertex $v$ of $\Gamma$ corresponds either to a Seifert fibered space or to a $3-$ manifold homeomorphic to (compact surface) $\times[0,1]$. This manifold is denoted by $M_{v}$ and called a vertex manifold.

(2) Each edge $e$ of $\Gamma$ corresponds either to a 3-manifold homeomorphic to $($ torus $) \times I$ or to a 3 -manifold homeomorphic to (annulus) $\times I$. This manifold is denoted by $M_{e}$ and called an edge manifold.

(3) If the edge manifold $M_{e}$ is homeomorphic to (torus) $\times I$ and $e$ is incident to a vertex $v$, then this incidence is realized by an identification of a boundary component of $M_{e}$ with a boundary component of $M_{v}$. In particular, $M_{v}$ must be Seifert fibered.

(4) If the edge manifold $M_{e}$ is homeomorphic to (annulus) $\times I$ and $e$ is incident to a vertex $v$, then this incidence is realized by an identification of a component of $(\partial$ (annulus $)) \times I$, with a subannulus of $\partial M_{v}$. If $M_{v}$ is Seifert fibered, then this subannulus of $\partial M_{v}$ consists of fibers of $M_{v}$. If $M_{v}$ is homeomorphic to (compact surface) $\times I$, then this subannulus is a component of $(\partial($ compact surface $)) \times I$.

(5) If a vertex manifold $M_{v}$ is homeomorphic to (compact surface) $\times I$, then the valence of $v$ equals the number of components of $(\partial$ (compact surface $)) \times I$. Ie, each component of $(\partial($ compact surface $)) \times I$ is identified with a subannulus of the boundary of an edge manifold.

A generalized graph manifold is totally orientable if each vertex manifold that is Seifert fibered is totally orientable and each vertex manifold that is not Seifert fibered is homeomorphic to (compact orientable surface) $\times I$.

The union of edge manifolds in $M$ is also called the characteristic submanifold of $M$. It is denoted by $\mathcal{E}$. The image of a torus or annulus, respectively, along which an indentification took place is called a decomposing torus or decomposing annulus, respectively. The union of decomposing tori and annuli is denoted by $\mathcal{T}$.

Consider the case in which an edge manifold $M_{e}=($ torus $) \times[0,1]$ of a graph manifold is cut along an incompressible torus $T=($ torus $) \times\{$ point $\}$. When $M_{e}$ is cut along $T$, the remnants of $M_{e}$ are (torus) $\times\left[0, \frac{1}{2}\right]$ and (torus) $\times\left[\frac{1}{2}, 1\right]$. Each of these remnants forms a collar of a vertex manifold. A foliation of (torus) by circles may be chosen in such a way that the Seifert fibration of the vertex manifold extends across the remnant. We may thus ignore these remnants, as we do in the above definition of generalized graph manifolds. This facilitates the discussion of strongly irreducible Heegaard splittings of generalized graph 
manifolds. Later, when considering amalgamations of strongly irreducible Heegaard splittings of generalized graph manifolds, we will have to reconsider these remnants.

Definition 4.4 A portion of the boundary of a vertex manifold $M_{v}$ of a generalized graph manifold $M$ that is contained in $\partial M$ is called an exterior boundary component of $M_{v}$. We denote the union of exterior boundary components of $M_{v}$ by $\partial_{E} M_{v}$.

\section{Motivational examples of Heegaard splittings}

In this section we describe some examples of Heegaard splittings for graph manifolds and generalized graph manifolds. The following definition facilitates describing the structure of certain surfaces.

Definition 5.1 Let $F$ be a surface in a 3-manifold $M$ and $\alpha$ an arc with interior in $M \backslash F$ and endpoints on $F$. Let $C(\alpha)$ be a collar of $\alpha$ in $M$. The boundary of $C(\alpha)$ consists of an annulus $A$ together with two disks $D_{1}, D_{2}$, which we may assume to lie in $F$. We call the process of replacing $F$ by $\left(F \backslash\left(D_{1} \cup D_{2}\right)\right) \cup A$ performing ambient 1 -surgery on $F$ along $\alpha$.

The process of ambient 1-surgery on a surface along an arc is sometimes informally referred to as "attaching a tube".

Example 5.2 Let $Q$ be a closed orientable surface. The standard Heegaard splitting of $Q \times \mathbb{S}^{1}$ may be constructed in more than one way. In particular, consider a small disk $D \subset Q$ and a collection $\Gamma$ of arcs that cut $Q \backslash D$ into a disk. Let $S$ be the result of performing ambient 1 -surgery on $\partial D \times \mathbb{S}^{1}$ along $\Gamma \times\{$ point $\}$. Then $S$ is the splitting surface of a Heegaard splitting $Q \times \mathbb{S}^{1}=V \cup_{S} W$ (for details, 20] ). See Figure 2, One of the handlebodies is $\left((\right.$ shaded disk $\left.) \times \mathbb{S}^{1}\right) \cup N($ dashed arcs $)$.

The same Heegaard splitting of $Q \times \mathbb{S}^{1}$ may be obtained in another way: Partition $\mathbb{S}^{1}$ into two intervals $I_{1}, I_{2}$ that meet in their endpoints. Consider two distinct points $p, q \in Q$. Then the surface obtained by performing ambient 1-surgery on $Q \times\left(I_{1} \cap I_{2}\right)$ along $\left(p \times I_{1}\right) \cup\left(q \times I_{2}\right)$ is isotopic to $S$ above.

The fact that this Heegaard splitting can be constructed either from a vertical torus or from horizontal surfaces via ambient 1-surgery is likely to be a very 


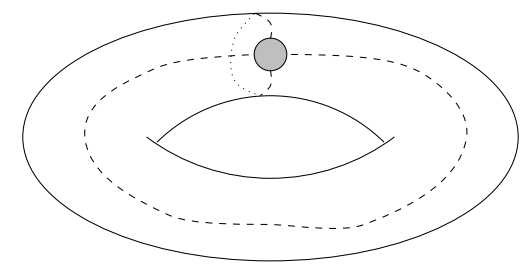

Figure 2: Schematic for Heegaard splitting of $Q \times \mathbb{S}^{1}$

special feature. But there are no general techniques for detecting the sort of global isotopies that allow this to happen.

Note also that this Heegaard splitting is not strongly irreducible. The two descriptions of this Heegaard splitting hint at distinct weak reductions. In one of these weak reductions, the incompressible surfaces would consist of vertical tori. In the other, the incompressible surfaces would consist of horizontal incompressible surfaces. In weak reductions of the latter type, we see that the Heegaard splitting is an amalgamation of two Heegaard splittings with mostly horizontal splitting surface.

The Heegaard splitting described turns out to be the only irreducible Heegaard splitting for a manifold of the form (closed orientable surface) $\times \mathbb{S}^{1}$. This fact is the main theorem of [20]. The first description of the construction can be generalized to Seifert fibered spaces to provide the canonical Heegaard splittings for totally orientable Seifert fibered spaces, see [1] and [13. The Heegaard splittings arising from this construction have been termed vertical. This terminology has created some confusion, because the splitting surface of a vertical Heegaard splitting is not vertical as a surface. Here we will continually focus on the splitting surface. In particular, we will want to distinguish between surfaces that are vertical and surfaces that are the splitting surface of a vertical Heegaard splitting. For this reason, we will augment the existing terminology and refer to the splitting surface of a vertical Heegaard splitting as "pseudovertical".

We recall the definition of a vertical Heegaard splitting for a Seifert fibered space. The structure of Heegaard splittings for totally orientable Seifert fibered spaces has been completely described in [13. Thus we may restrict our attention to Seifert fibered spaces with non empty boundary in the definition below.

Definition 5.3 Let $M$ be a Seifert fibered space with $\partial M \neq \emptyset$. Denote the base orbifold of $M$ by $O$. Denote the exceptional fibers of $M$ by $f_{1}, \ldots, f_{n}$ and the corresponding exceptional points in $O$ by $e_{1}, \ldots, e_{n}$. Denote the boundary 


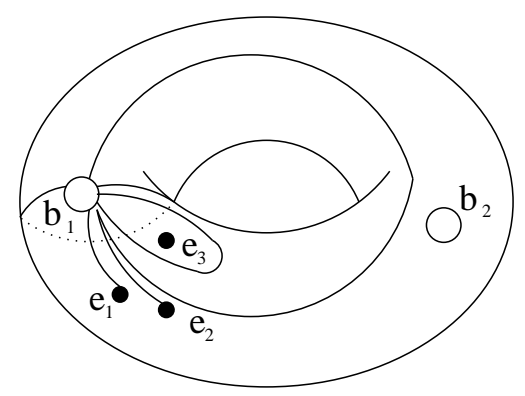

Figure 3: Schematic for a vertical Heegaard splitting of a Seifert fibered space

components of $M$ by $B_{1}, \ldots, B_{m}$ and the corresponding boundary components of $O$ by $b_{1}, \ldots, b_{m}$,

Partition $f_{1}, \ldots, f_{n}$ into two subsets: $f_{1}, \ldots, f_{i}$, the fibers that will lie in $V$ and $f_{i+1}, \ldots, f_{n}$, the fibers that will lie in $W$. Then partition $B_{1}, \ldots, B_{m}$ into two subsets: $B_{1}, \ldots, B_{j}$, the boundary components that will lie in $V$ and $B_{j+1}, \ldots, B_{m}$, the boundary components that will lie in $W$.

We may assume that $j \geq 1$, for otherwise we may interchange the roles of $V$ and $W$ in the construction below. Let $\Gamma$ be a collection of $\operatorname{arcs}$ in $O$ each with at least one endpoint on $b_{1}$ such that $O \backslash \Gamma$ is a regular neighborhood of $e_{i+1} \cup \cdots \cup e_{n} \cup b_{j+1} \cup \cdots \cup b_{m}$ or of a point, if this set is empty. See Figure 3,

Set $V=N\left(f_{1} \cup \cdots \cup f_{i} \cup B_{1} \cup \cdots \cup B_{j} \cup \Gamma\right)$ and set $W=\operatorname{closure}(M \backslash V)$. Set $S=\partial_{+} V=\partial_{+} W$. Then $M=V \cup_{S} W$ is a Heegaard splitting. (For details, see [21] or [13.) A Heegaard splitting of a Seifert fibered space with non empty boundary constructed in this manner is called a vertical Heegaard splitting. A pseudovertical surface is a surface that is the splitting surface of a vertical Heegaard splitting.

A little more work is required to extend this notion to the setting of graph manifolds. Here we consider the intersection of a Heegaard splitting of a generalized graph manifold with a vertex manifold that is a Seifert fibered space. Denote the Heegaard splitting by $M=V \cup_{S} W$ and the vertex manifold by $M_{v}$. Here $S \cap M_{v}$ is not necessarily connected. Furthemore, $S \cap M_{v}$ has boundary. A concrete description of all possible such surfaces would be quite extensive. For this reason, we give the following, purely structural, definition:

Definition 5.4 Let $M=V \cup_{S} W$ be a Heegaard splitting of a generalized graph manifold with non empty characteristic submanifold. Let $M_{v}$ be a Seifert 
fibered vertex manifold of $M$. We say that $S \cap M_{v}$ is pseudovertical if the following holds:

There is a collection of vertical annuli and tori $\mathcal{A} \subset M_{v}$. And there is a collection of arcs $\Gamma$ in the interior of $M_{v}$ such that each endpoint of each arc in $\Gamma$ lies in $\mathcal{A}$ and such that $\Gamma$ projects to a collection of disjoint imbedded arcs in $O_{v}$. And $S \cap M_{v}$ is obtained from $\mathcal{A}$ by ambient 1-surgery along $\Gamma$.

The assumption that $M=V \cup_{S} W$ is a Heegaard splitting places strong restrictions on $S$. If $S \cap M_{v}$ is pseudovertical, then $S \cap\left(\partial M_{v} \backslash \partial_{E} M_{v}\right)$ consists of vertical curves. Thus $V \cap\left(\partial M_{v} \backslash \partial_{E} M_{v}\right)$ and $W \cap\left(\partial M_{v} \backslash \partial_{E} M_{v}\right)$ consist of annuli. Each such annulus is either a spanning annulus in $V$ or $W$, or it has both boundary components in $S$.

Consider the result of cutting a compression body $V$ along an annulus $A$ with $\partial A \subset \partial_{+} V$. If the annulus is inessential, then the effect is nil. If the annulus is essential, then the result is again a, possibly disconnected, compression body, see [23. Lemma 2]. If the annulus is a spanning annulus, then the result is a handlebody. But in this context we should think of it as a compact 3 manifold of the form $(($ compact surface $) \times I) \cup(1$ - handles $)$. The only way this can happen, given the structure of $S \cap M_{v}$, is if the components of $V \cap M_{v}$ and $W \cap M_{v}$ are constructed from vertical solid tori and perhaps components homeomorphic to (annulus) $\times \mathbb{S}^{1}$ by attaching "horizontal" 1 -handles. For more concrete computations, see [24].

It is a non trivial fact that for Seifert fibered spaces the two definitions of pseudovertical surfaces coincide. This follows from [20, Proposition 2.10] via Lemma 7.1 (an adaptation of the central argument in [21]) along with Lemma 7.4. For an illustration, see the final remarks in the example below.

Example 5.5 Let $M$ be a Seifert fibered space with base orbifold a disk and with two exceptional fibers $f_{1}, f_{2}$. Let $T$ be a boundary parallel torus and let $\alpha$ be an arc connecting $T$ to itself that projects to an imbedded arc that separates the two exceptional points. Let $S$ be the result of performing ambient 1 -surgery on $T$ along $\alpha$. Then $S$ is the splitting surface of a Heegaard splitting of $M=V \cup_{S} W$ (see [13]).

Now consider two copies $M_{1}, M_{2}$ of $M$ with Heegaard splittings $M_{i}=V_{i} \cup_{S_{i}} W_{i}$. We may assume that $\partial M_{1} \subset V_{1}$ and $\partial M_{2} \subset W_{2}$. We identify $\partial M_{1}$ and $\partial M_{2}$ to obtain a $3-$ manifold $\tilde{M}$. Juxtaposing the two Heegaard splittings provides a generalized strongly irreducible Heegaard splitting. It is indicated schematically in Figure 4 Amalgamating the two Heegaard splittings along $\partial M_{1}, \partial M_{2}$ results 
in a Heegaard splitting $\tilde{M}=V \cup_{S} W$. The result is schematically indicated in Figure 5] The circles correspond to vertical tori. The splitting surfaces of the Heegaard splittings are obtained by performing ambient 1-surgery on these tori along arcs in $\tilde{M}$ corresponding to the dashed arcs.

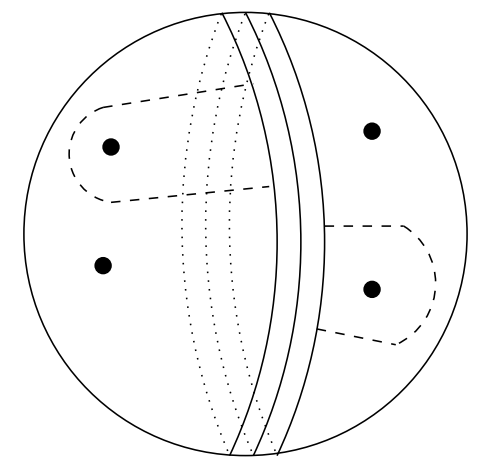

Figure 4: Schematic for a generalized strongly irreducible Heegaard splitting

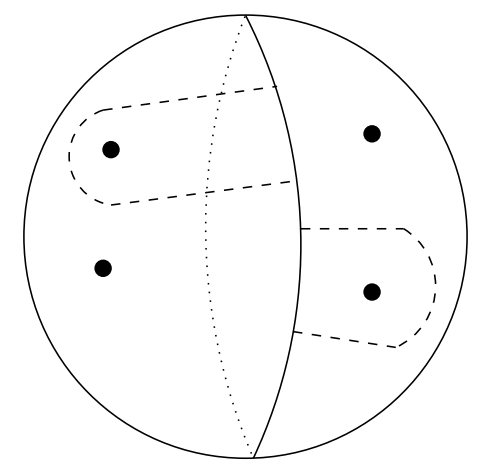

Figure 5: Schematic for Heegaard splitting after amalgamation

The manifold $\tilde{M}$ obtained when $\partial M_{1}$ and $\partial M_{2}$ are identified is a graph manifold modelled on a graph with two vertices and one edge connecting the two vertices. The vertex manifolds are slightly shrunken versions of $M_{1}$ and $M_{2}$. The edge manifold is a collar of the image of $\partial M_{1}$ and $\partial M_{2}$ in $\tilde{M}$.

If the homeomorphism that identifies $\partial M_{1}$ with $\partial M_{2}$ is fiberpreserving, then $\tilde{M}$ is in fact a Seifert fibered space. In this case $S$ is isotopic to the surface indicated schematically in Figure 6. This surface is obtained by performing ambient 1-surgery along two arcs corresponding to the dashed arcs on the two vertical tori corresponding to the two solid circles. 


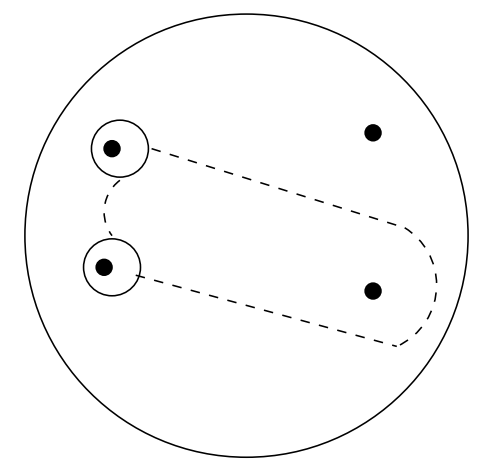

Figure 6: Schematic for Heegaard splitting of a Seifert fibered space

The above construction can be generalized to arbitrary graph manifolds. The resulting Heegaard splittings can be considered the canonical Heegaard splittings and generalized Heegaard splittings of graph manifolds. Contemplation of Figure 5 might lead one to believe that amalgamations of strongly irreducible Heegaard splittings still have enough structure to be described in the terminology used here. But this is not the case, as we shall see in the following example.

Example 5.6 Let $M_{1}$ be as above. Let $P$ be a thrice punctured $\mathbb{S}^{2}$. Denote the boundary components of $P$ by $b_{1}, b_{2}, b_{3}$. Let $M_{2}$ be the $3-$ manifold obtained from $P \times \mathbb{S}^{1}$ by identifying $b_{2} \times \mathbb{S}^{1}$ and $b_{3} \times \mathbb{S}^{1}$ via a homeomorphism that takes $b_{2} \times\{$ point $\}$ to $\{$ point $\} \times \mathbb{S}^{1}$.

Here $M_{2}$ is a graph manifold modelled on a graph with one vertex and one edge. It has a Heegaard splitting depicted schematically in Figure 7 In $P \times \mathbb{S}^{1}$, we take the products of the regions pictured. This yields a white (annulus) $\times \mathbb{S}^{1}$ and a shaded solid torus. When $b_{2} \times \mathbb{S}^{1}$ and $b_{3} \times \mathbb{S}^{1}$ are identified, both (annulus) $\times \mathbb{S}^{1}$ and the solid torus meet themselves in (square) disks. Thus we obtain a (strongly irreducible) Heegaard splitting of genus 2 for $M_{2}$. Denote the compression body by $V_{2}$ and the handlebody by $W_{2}$.

This Heegaard splitting can also be constructed by ambient 1-surgery on a boundary parallel torus along an arc as pictured in Figure 8.

The description indicated schematically in Figure 7 is preferable to the one indicated schematically in Figure 8. This is because the splitting surface of the former is vertical in the Seifert fibered vertex manifold and has a very special structure in the edge manifold. The latter intersects the edge manifold in a tube. 


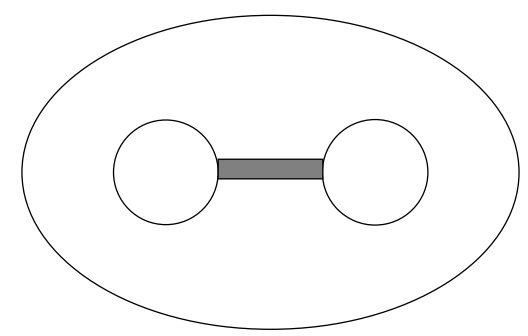

Figure 7: Schematic for Heegaard splitting of a graph manifold

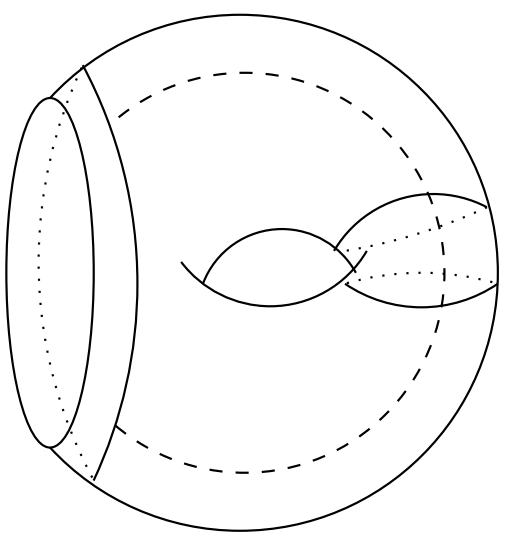

Figure 8: Schematic for Heegaard splitting of a graph manifold

Now let $M$ be a 3-manifold obtained by identifying the boundary component of $M_{1}$ to the boundary component of $M_{2}$. Then $M$ is a graph manifold modelled on a graph as in Figure 9

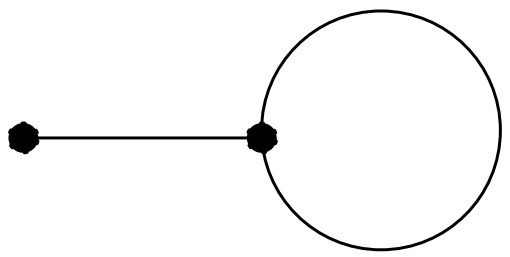

Figure 9: The graph on which $M$ is modelled

Here $M$ inherits a strongly irreducible generalized Heegaard splitting that is the juxtaposition of the strongly irreducible Heegaard splittings of $M_{1}$ and $M_{2}$. If we wish to amalgamate the two Heegaard splittings, recall that the collar neighborhood of $\partial M_{2}$ that lies in $V_{2}$ will be identified to a single torus. 
To prevent this identification from interfering with the decomposing tori of $M_{2}$, we are forced to consider the second description of the Heegaard splitting $M_{2}=V_{2} \cup_{S_{2}} W_{2}$. But this means that our description of the resulting Heegaard splitting involves a tube which runs through an edge manifold.

The tube in the above description of the Heegaard splitting does not fit nicely into the characterization of Heegaard splittings as horizontal, pseudohorizontal, vertical or pseudovertical. The moral is, that such tubes arise and can be quite complicated. A more thorough investigation of the nature of such tubes will be the subject of a further investigation. However, such tubes do not arise in strongly irreducible Heegaard splittings. For this reason we prefer to think of our Heegaard splittings as amalgamations of strongly irreducible Heegaard splittings.

The following two examples illustrate more peculiar Heegaard splittings that arise under special circumstances.

Example 5.7 Let $N$ be a Seifert fibered space with base orbifold the sphere and with four exceptional fibers $f_{1}, \ldots, f_{4}$ with carefully chosen invariants: $\frac{1}{2}, \frac{1}{2}, \frac{1}{2}, \frac{l}{2 l+1}$. Here $N \backslash \eta\left(f_{4}\right)$ is a Seifert fibered manifold with boundary and hence fibers over the circle. More specifically, it fibers as a once punctured torus bundle over the circle. By partitioning the circle into two intervals $I_{1}, I_{2}$ that meet in their endpoints, we obtain a decomposition $N \backslash \eta\left(f_{4}\right)=V^{\prime} \cup_{S^{\prime}} W^{\prime}$ with $V^{\prime}=($ once punctured torus $) \times I_{1}, W^{\prime}=($ once punctured torus $) \times I_{2}$ and $S^{\prime}=$ two once punctured tori.

Note that this decomposition is not a Heegaard splitting in the sense used here as $S^{\prime}$ is not closed. Now the carefully chosen invariants guarantee that the boundary of a meridian disk of $N\left(f_{4}\right)$ meets $\partial V^{\prime}$ in a single arc. In particular, $V=V^{\prime} \cup N\left(f_{4}\right)$ is also a handlebody (of genus two). Setting $W=W^{\prime}$, this defines a Heegaard splitting $N=V \cup_{S} W$. The splitting surface of this Heegaard splitting is a horizontal surface away from $N\left(f_{4}\right)$. And after a small isotopy, $S \cap N\left(f_{4}\right)$ is a collar of $f_{4}$. Thus $S$ is pseudohorizontal.

Example 5.8 Let $Q$ be a once punctured torus. Set $M_{i}=Q \times \mathbb{S}^{1}$ for $i=1,2$. Partition $\mathbb{S}^{1}$ into two intervals $I_{1}, I_{2}$ meeting in their endpoints. Set $V_{i}=$ $Q \times I_{1} \subset M_{i}$ and $W_{i}=Q \times I_{2} \subset M_{i}$. Let $T_{i}=\partial M_{i}$ and $c_{i}=\partial Q \times\{$ point $\} \subset T_{i}$. Identify $T_{1}$ and $T_{2}$ via a homeomorphism so that $\left|c_{1} \cap c_{2}\right|=1$. Then $V_{1}$ and $V_{2}$ meet in a (square) disk, hence $V=V_{1} \cup V_{2}$ is a handlebody. Similarly, $W=W_{1} \cup W_{2}$ is a handlebody. Set $S=\partial V=\partial W$, then $M=V \cup_{S} W$ is a Heegaard splitting of $M=M_{1} \cup_{T_{1}=T_{2}} M_{2}$. 
Here $M$ is a graph manifold modelled on a graph with two vertices and one edge connecting the two vertices. Its characteristic submanifold is a collar of $T_{1}=T_{2}$. In the vertex manifolds, $S$ is horizontal. In the edge manifold, $S$ has a very specific structure.

Definition 5.9 Let $M$ be a generalized graph manifold with characteristic submanifold $\mathcal{E}$. Let $M=V \cup_{S} W$ be a Heegaard splitting. We say that $M=$ $V \cup_{S} W$ is standard if $S$ can be isotoped so that for each vertex manifold $M_{v}$ of $M, S \cap M_{v}$ is either horizontal, pseudohorizontal, vertical or pseudovertical and such that for each edge manifold $M_{e}$ of $M, S \cap M_{e}$ is characterized by one of the following:

(1) $S \cap M_{e}$ is a collection of incompressible annuli (including spanning annuli and possibly boundary parallel annuli) or is obtained from such a collection by ambient 1-surgery along an arc which is isotopic into $\partial M_{e}$.

(2) $M_{e}$ is homeomorphic to (torus) $\times I$ and there is a pair of simple closed curves $c, c^{\prime} \subset$ (torus) such that $c \cap c^{\prime}$ consists of a single point $p \in$ (torus) and either $V \cap(($ torus $) \times I)$ or $W \cap(($ torus $) \times I)$ is a collar of $(c \times\{0\}) \cup(p \times I) \cup$ $\left(c^{\prime} \times\{1\}\right)$.

\section{The active component}

In this section we consider a generalized graph manifold $W$. We show that if $M=V \cup_{S} W$ is a strongly irreducible Heegaard splitting, then $S$ may be isotoped so that it is incompressible away from a single vertex or edge manifold of $M$.

Lemma 6.1 Let $M$ be a generalized graph manifold with characteristic submanifold $\mathcal{T}$. Let $M=V \cup_{S} W$ be a strongly irreducible Heegaard splitting. Let $\mathcal{D}_{V}$ be a collection of defining disks for $V$ and $\mathcal{D}_{W}$ a collection of defining disks for $W$. There is a vertex or edge manifold $N$ of $M$ so that, after isotopy, each outermost disk component of both $\mathcal{D}_{V} \backslash\left(\mathcal{T} \cap \mathcal{D}_{V}\right)$ and of $\mathcal{D}_{W} \backslash\left(\mathcal{T} \cap \mathcal{D}_{W}\right)$ lies in $N$. Moreover, for each vertex or edge manifold $\tilde{N} \neq N, S \cap \tilde{N}$ is incompressible.

Proof Isotope $S$ so that $\mathcal{T} \cap S$ consists only of curves essential in both $\mathcal{T}$ and $S$. Furthermore, assume that the isotopy has been chosen so that $|\mathcal{T} \cap S|$ is minimal subject to this condition. Suppose that $D^{\prime}$ is an outermost subdisk of $\mathcal{D}_{V} \backslash(\mathcal{T} \cap \mathcal{D})$. Let $N$ be the vertex or edge manifold of $M$ containing $D^{\prime}$. Then either $D^{\prime}$ is a disk in the interior of $V$ or $D^{\prime}$ meets an annular component $A$ 
of $V \cap \partial N$. In case of the latter, $\partial D^{\prime}$ meets $A$ in a single arc, $a$. Let $D^{\prime \prime}$ be the disk obtained by cutting $A$ along $a$, adding two copies of $D^{\prime}$ and isotoping the result to be a properly imbedded disk in $V$. The assumption that $|\mathcal{T} \cap S|$ be minimal guarantees that $D^{\prime \prime}$ is an essential disk in $V$. Thus in both cases, there is an essential disk properly imbedded in $V$ that lies in the interior of $N$, we refer to this disk as $D$.

Similarly, consider an outermost subdisk of $\mathcal{D}_{W} \backslash\left(\mathcal{T} \cap \mathcal{D}_{W}\right)$. The above argument shows that in a vertex or edge manifold $N^{\prime}$ of $M$, there is an essential disk $E$ properly imbedded in $W$. Since $M=V \cup_{S} W$ is strongly irreducible, $D$ must meet $E$, hence the vertex or edge manifold $N^{\prime}$ must coincide with the vertex or edge manifold $N$.

It follows that for each vertex or edge manifold $\tilde{N} \neq N, S \cap \tilde{N}$ is incompressible.

Definition 6.2 If $M$ is a generalized graph manifold, with a strongly irreducible Heegaard splitting $M=V \cup_{S} W$, then the vertex or edge manifold $N$ as in Lemma 6.1 is called the active component of $M=V \cup_{S} W$.

\section{What happens in the active component?}

The possibilities for the active component depend on the type of the active component. There are five possibilities. We discuss each in turn.

\subsection{Seifert fibered vertex manifold with exterior boundary}

We first consider the case in which the active component of $M=V \cup_{S} W$ is a vertex manifold $M_{v}$ that is a Seifert fibered space and that has exterior boundary. This situation has been studied extensively in the more restricted case in which $M$ itself is a Seifert fibered space.

Lemma 7.1 Suppose $M$ is a totally orientable Seifert fibered space with non empty boundary and $M=V \cup_{S} W$ is a Heegaard splitting. Then each exceptional fiber of $M_{v}$ is a core of either $V$ or $W$.

Proof This is [21, Lemma 4.1], the central argument in [21]. 
Theorem 7.2 Suppose $M$ is a totally orientable Seifert fibered space with non empty boundary. Suppose $M=V \cup_{S} W$ is a Heegaard splitting. Then $S$ is pseudovertical.

Proof This is the main theorem of [21].

The argument extends to a more general setting. Indeed, the isotopy performed in [21, Lemma 4.1] takes place within a small regular neighborhood of a saturated annulus. In particular, the isotopy can here be performed entirely within $M_{v}$. The only requirements on this saturated annulus are that one boundary component has to lie on the splitting surface of the Heegaard splitting and the other has to wrap around the exceptional fiber.

Lemma 7.3 Suppose that $M$ is a totally orientable graph manifold and $M=$ $V \cup_{S} W$ is a Heegaard splitting. Suppose further that there is an exceptional fiber $f$ in $M_{v}$ and an annulus $A$ such that:

(1) One component of $\partial A$ wraps at least twice around $f$; and

(2) $A$ is embedded away from $\partial A \cap f$; and

(3) $\partial A \backslash f$ lies in $S$.

Then $f$ is a core of either $V$ or $W$.

Proof In fact, in the central argument in [21], the existence of a boundary component is used exclusively to produce such an annulus.

This more general lemma will be used in the next subsection. A consequence of Lemma 7.1 is that we can make use of the following lemma.

Lemma 7.4 Suppose that $f$ is an exceptional fiber of $M_{v}$ and that $f$ is also a core of $V$. Then $M \backslash \eta(f)=(V \backslash \eta(f)) \cup_{S} W$ is a Heegaard splitting. Furthermore, $S \cap\left(M_{v} \backslash \eta(f)\right)$ is vertical or pseudovertical, respectively, if and only if $S \cap M_{v}$ is vertical or pseudovertical, respectively. The same holds if $f$ is a core of $W$.

Proof Since $f$ is a core of $V, V \backslash \eta(f)$ is still a compression body. Thus $M \backslash \eta(f)=(V \backslash \eta(f)) \cup_{S} W$ is a Heegaard splitting. Conversely, if $M \backslash \eta(f)=$ $(V \backslash \eta(f)) \cup_{S} W$ is a Heegaard splitting, then $(V \backslash \eta(f)) \cup N(f)$ is a compression body. Hence $M=V \cup_{S} W$ is a Heegaard splitting.

Now by the definition of vertical and pseudovertical, respectively, $S \cap\left(M_{v} \backslash \eta(f)\right)$ is vertical or pseudovertical, respectively, if and only if $S \cap M_{v}$ is vertical or pseudovertical, respectively. Compare Figures [6] and 10. 


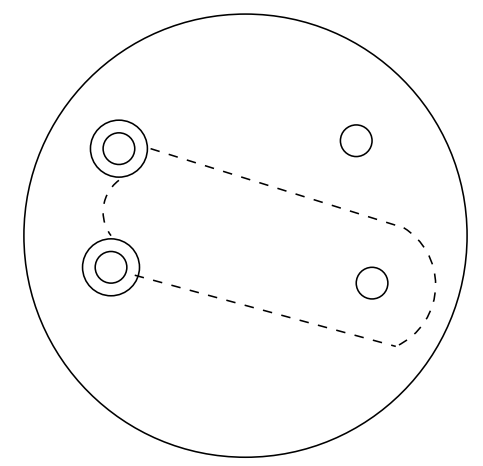

Figure 10: Schematic for vertical Heegaard splittings of graph manifold with boundary

The following Proposition generalizes Theorem [7.2, But because Theorem $\mathbf{7 . 2}$ is already known, we restrict our attention to the case in which $M$ has a non empty characteristic submanifold.

Proposition 7.5 Suppose that $M$ is a connected generalized graph manifold with non empty characteristic submanifold. Suppose that $M=V \cup_{S} W$ is a strongly irreducible Heegaard splitting. Suppose that the active component of $M=V \cup_{S} W$ is a vertex manifold $M_{v}$ that is a Seifert fibered space and that has exterior boundary. Then we may isotope the decomposing tori, thereby redefining $M_{v}$ and the edge manifolds for which $e$ is incident to $v$ slightly, so that after this isotopy, $S \cap M_{v}$ is a vertical surface and so that an edge manifold becomes the active component.

Proof The proof is by induction on the number of exceptional fibers in $M_{v}$. Suppose first that $M_{v}$ contains no exceptional fibers. Denote the exterior boundary of $M_{v}$ by $\partial_{E} M_{v}$. Let $\mathcal{A}$ be a collection of disjoint essential vertical annuli in $M_{v}$ that cut $M_{v}$ into a regular neighborhood of $\partial M_{v} \backslash \partial_{E} M_{v}$. After an isotopy, $S \cap \mathcal{A}$ consists of closed curves essential in both $S$ and $\mathcal{A}$ and in the minimal possible number of such curves. In particular, after a small isotopy, this intersection consists of regular fibers of $M_{v}$.

Now isotope $S$ so that $S \cap N(\mathcal{A})$ consists of vertical incompressible annuli. Set $\tilde{M}_{v}=N\left(\partial_{E} M_{v}\right) \cup N(\mathcal{A})$. Isotope $S$ so that $S \cap \tilde{M}_{v}$ consists of $S \cap N(\mathcal{A})$ together with annuli in $N\left(\partial_{E} M_{v}\right) \backslash N(\mathcal{A})$ that join two components of $S \cap N(\mathcal{A})$. Isotope any annuli in $S \cap\left(M_{v} \backslash \tilde{M}_{v}\right)$ that are parallel into $\partial \tilde{M}_{v}$ into $\tilde{M}_{v}$. Set $\partial_{E} \tilde{M}_{v}=\partial_{E} M_{v}$.

Let $\tilde{T}$ be a component of $\partial \tilde{M}_{v} \backslash \partial_{E} \tilde{M}_{v}$. Then $\tilde{T}$ is parallel to a decomposing torus or annulus $T$. We replace $T$ by $\tilde{T}$. We may do so via an isotopy. We do 


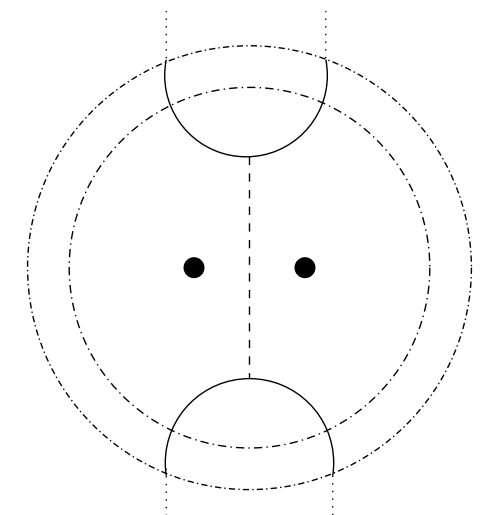

Figure 11: The active component is a vertex manifold

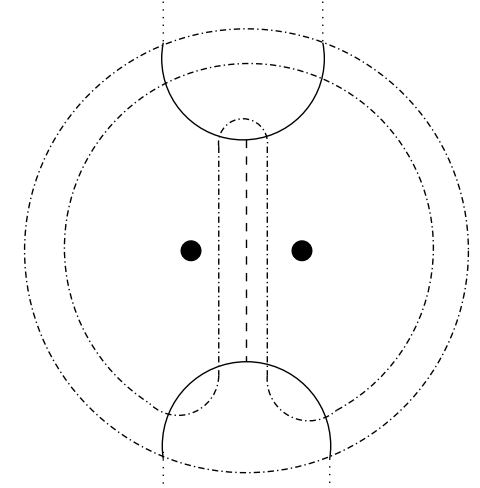

Figure 12: The active component is an edge manifold

this for all components of $\partial \tilde{M}_{v} \backslash \partial_{E} \tilde{M}_{v}$. After this process, the conclusions of the proposition hold. See Figures [11] and 12

To prove the inductive step, suppose that $f$ is an exceptional fiber of $M_{v}$. Then by Lemma 7.1 $f$ is a core of either $V$ or $W$, say of $V$. The inductive hypothesis in conjunction with Lemma 7.4 then proves the theorem.

\subsection{Seifert fibered vertex manifold without exterior boundary}

Next we consider the case in which the active component of $M=V \cup_{S} W$ is a vertex manifold $M_{v}$ that is a Seifert fibered space and that has no exterior boundary. Part of the strategy is somewhat reminiscent of the strategy used in 
the preceding case. However, the situation here is more complicated and a more refined strategy must be used. The refined strategy involves a generalization of Lemma 3.11 to a "spine" of the vertex manifold.

Definition 7.6 Let $Q_{v}$ be the base orbifold of $M_{v}$. A spine for $Q_{v}$ is a 1complex $\Gamma^{1}$ with exactly one vertex $v$ that cuts $Q_{v}$ into a regular neighborhood of $\partial Q_{v} \cup($ exceptional points $)$. A 2-complex of the form $\Gamma^{2}=p^{-1}\left(\Gamma^{1}\right)$ is called a spine of $M_{v}$. We denote the regular fiber $p^{-1}(v)$ by $\gamma$.

The following lemma generalizes Lemma 3.11. Though we will only be interested in this lemma in the case that $N$ is a vertex manifold of a graph manifold, we state it in very general terms. It applies to a larger class of 3-manifolds than just graph manifolds.

Lemma 7.7 Let $M=V \cup_{S} W$ be a strongly irreducible Heegaard splitting. Let $N$ be a totally orientable Seifert fibered submanifold of $M$ that doesn't meet $\partial M$. Let $\Gamma^{2}$ be a spine of $N$. Then $S$ may be isotoped so that the following hold:

(1) $S \cap \Gamma^{2}$ consists of simple closed curves and simple closed curves wedged together at points in $\gamma$.

(2) No closed curve in $S \cap \Gamma^{2}$ bounds a disk in $\Gamma^{2} \backslash S$.

Proof The first part of the assertion follows by general position. To prove the second assertion, let $X$ be a spine of $V$ and $Y$ a spine of $W$. Then $M \backslash\left(\partial_{-} V \cup X \cup \partial_{-} W \cup Y\right)$ is homeomorphic to $S \times(0,1)$. $X$ can't be disjoint from $\Gamma^{2}$. Thus for $t$ near $0,(S \times t) \cap \Gamma^{2}$ contains simple closed curves that bound essential disks in $V . Y$ can't be disjoint from $\Gamma^{2}$ either. Thus for $t$ near 1, $(S \times t) \cap \Gamma^{2}$ contains simple closed curves that bound essential disks in $W$.

As $t$ increases, $(S \times t) \cap \Gamma^{2}$ changes continuously. Since $M=V \cup_{S} W$ is strongly irreducible, there can be no $t$ such that $(S \times t) \cap \Gamma^{2}$ contains simple closed curves that bound essential disks in $V$ and simple closed curves that bound essential disks in $W$. Thus, there is a $t_{0}$, such that $\left(S \times t_{0}\right) \cap \Gamma^{2}$ contains no simple closed curves that bound essential disks in $V$ or $W$. Any remaining disk components in $\left(S \times t_{0}\right) \cap \Gamma^{2}$ must be inessential in $V$ or $W$ and can hence be removed via isotopy. The lemma follows.

Before launching into the two main portions of the argument, we prove an auxiliary lemma. This lemma is a weak version of a counterpart to Theorem 3.3 in [17]. 


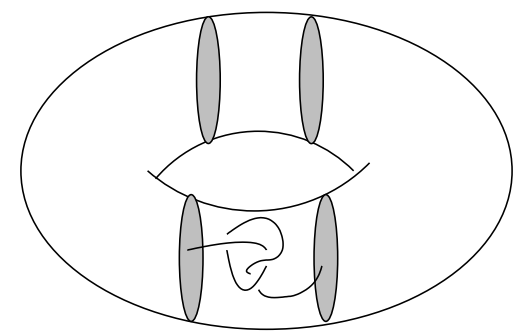

Figure 13: Schematic for a Heegaard splitting intersecting a solid torus

Lemma 7.8 Suppose $M=V \cup_{S} W$ is a strongly irreducible Heegaard splitting of a 3-manifold $M$. Suppose $U \subset M$ is a solid torus such that $S$ intersects $\partial U$ in meridians. Further suppose that $\partial(M \backslash$ interior $(U))$ is incompressible in $M \backslash$ interior $(U)$. Then $S \cap U$ consists of meridian disks of $U$ and components that are obtained by ambient 1-surgery on pairs of meridian disks along a single arc that joins the two meridian disks.

Proof Let $s$ be a component of $S \cap \partial U$. A collar of $s$ in $S$ is an annulus $A$. Lemma 2.6 in [19] states: "Suppose $S$ gives a Heegaard splitting of a 3manifold $M$ into compression bodies $V$ and $W$. Suppose that $F \subset S$ is a compact subsurface so that every component of $\partial F$ is essential in $S$. Suppose each component of $\partial F$ bounds a disk in $M$ disjoint from interior $(F)$. Either $\partial F$ bounds a collection of disks in a single compression body or $M=V \cup_{S} W$ is weakly reducible."

Here $M=V \cup_{S} W$ is strongly irreducible. It follows that either $s$ bounds a disk in $S$ or $s$ bounds a disk in a single compression body, say $V$. Here $\partial(M \backslash$ interior $(U))$ is incompressible in $M \backslash$ interior $(U)$. It follows that in case of the former, $s$ bounds a meridian disk of $U$ in $S \cap U$ and that in case of the latter, a meridian disk of $U$ bounded by $s$ lies entirely in $V$.

Thus either $s$ bounds a meridian disk in $S \cap U$ or bounds a meridian disk in the surface obtained by compressing $S \cap U$ along $D$. It follows that $S \cap U$ may be reconstructed from meridian disks by ambient 1-surgery along a collection of arcs such that each meridian disk meets at most one endpoint of one of the arcs. In particular, between two meridian disks there is at most one arc.

Lemma 7.9 Suppose that the active component of $M=V \cup_{S} W$ is a vertex manifold $M_{v}$ that is a Seifert fibered space with boundary but no exterior boundary. Then one of the following holds: 
(1) We may isotope the decomposing tori, thereby redefining $M_{v}$ and the edge manifolds for which $e$ is incident to $v$ slightly, so that after this isotopy, $S \cap M_{v}$ is either a horizontal or vertical incompressible surface and so that an edge manifold becomes the active component.

Or:

(2) $S$ may be isotoped within $M_{v}$ so that a fiber $f$ of $M_{v}$ lies in $S$.

Proof Let $\Gamma^{2}$ be a spine of $M_{v}$ and isotope $S$ within $M_{v}$ so that the conclusions of Lemma 7.7 hold. Three cases need to be considered:

Case 1 A simple closed curve in $S \cap \Gamma^{2}$ can be isotoped to be vertical.

Then $S$ may be isotoped in $M_{v}$ so that it contains a regular fiber of $M_{v}$.

Case 2 No simple closed curve in $S \cap \Gamma^{2}$ can be isotoped to be vertical and in each solid torus component $U$ of $M_{v} \backslash \eta\left(\Gamma^{2}\right), S \cap \partial U$ consists of meridians.

In this case, after a small isotopy, $S \cap N\left(\Gamma^{2}\right)$ is a horizontal incompressible surface. Furthermore, let $U$ be a component of $M_{v} \backslash \eta\left(\Gamma^{2}\right)$. By Lemma 7.8 $S \cap U$ consists of meridian disks possibly together with other components that are obtained by ambient 1-surgery on pairs of meridian disks along a single arc that joins the two meridian disks. Isotope each such arc out of $U$, through $N\left(\Gamma^{2}\right)$, avoiding $\gamma$, to lie in a component of $N\left(\partial M_{v}\right)$. Then $S \cap U$ consists of meridians for each solid torus component of $M_{v} \backslash \Gamma^{2}$ and all ambient 1-surgeries occur in $N\left(\partial M_{v}\right)$.

Let $\tilde{M}_{v}$ be the union of $N\left(\Gamma^{2}\right)$ with the solid tori containing the exceptional fibers of $M_{v}$. Then $\tilde{M}_{v}$ is a shrunk version of $M_{v}$. Note that $S \cap \tilde{M}_{v}$ is a horizontal incompressible surface. Let $\tilde{T}$ be a component of $\partial \tilde{M}_{v}$. Then $\tilde{T}$ is parallel to a decomposing torus $T$. We replace $T$ by $\tilde{T}$. We may do so via an isotopy. We do this for all components of $\partial \tilde{M}_{v}$. After this process, the conclusions of the lemma hold.

Case 3 No simple closed curve in $S \cap \Gamma^{2}$ can be isotoped to be vertical and there is a solid torus component $U$ of $M_{v} \backslash \eta\left(\Gamma^{2}\right)$ such that $S \cap \partial U$ does not consist of meridians.

The argument in this case is given, for instance, in Proposition 1.1 of [2] For completeness we povide a sketch of an argument more in line with the ideas used here. In this case there is a possibly singular annulus $A$ between a component of $S \cap \partial U$ and the exceptional fiber $f$ in $U$. If $A$ is not singular, then $A$ describes an isotopy of $S$ after which $f$ lies in $S$. If $A$ is singular, then $A$ satisfies the hypotheses of Lemma 7.3. Thus $f$ is a core of either $V$ or $W$. 
Ie, $S$ is the splitting surface for a Heegaard splitting of $M \backslash \eta(f)$. But then Proposition [7.5 applies to $M_{v} \backslash \eta(f)$ and we may isotope the decomposing tori, thereby redefining $M_{v}$ and the edge manifolds for which $e$ is incident to $v$ slightly, so that after this isotopy, $S \cap M_{v}$ is a vertical incompressible surface and so that an edge manifold becomes the active component.

Lemma 7.10 Suppose that the active component of $M=V \cup_{S} W$ is a vertex manifold $M_{v}$ that is a Seifert fibered space with boundary but no exterior boundary. Suppose further that a fiber $f$ of $M_{v}$ lies in $S$. Then one of the following holds:

(1) We may isotope the decomposing tori, thereby redefining the active component $M_{v}$ and the edge manifolds for which $e$ is incident to $v$ slightly, so that after this isotopy, $S \cap M_{v}$ is a vertical incompressible surface and so that an edge manifold becomes the active component.

Or:

(2) $S \cap M_{v}$ is pseudohorizontal.

Proof Consider a small regular neighborhood $N(f)$ of $f$ such that $S \cap N(f)$ is a collar $A$ of $f$. Compress $S$ as much as possible in $M_{v} \backslash \eta(f)$ to obtain an incompressible surface $S^{*} \subset M \backslash \eta(f)$. By Haken's Theorem, each compressing disk can be chosen to lie entirely on one side of $S$. Since $M=V \cup_{S} W$ is strongly irreducible, all compressions must have been performed to one side of $S$. It follows that $S^{*}$ lies either in $V$ or in $W$. There are three options for $S^{*} \cap M_{v}$ :

Case $1 S^{*} \cap\left(M_{v} \backslash \eta(f)\right)$ contains an annulus that is parallel into $\partial N(f)$.

Note that $S \cap N(f)$ is also parallel into $\partial N(f)$. Thus $S \cap M_{v}=\left(S^{*} \cap\right.$ $\left.\left(M_{v} \backslash \eta(f)\right)\right) \cup(S \cap N(f))$ contains a torus bounding a solid torus in either $V$ or $W$. Furthermore, $f$ lies on the boundary of this solid torus and meets a meridian disk once. After a small isotopy, $f$ is a core of the solid torus. Thus $f$ is a core of either $V$ or $W$. But then Proposition 7.5 applies to $M_{v} \backslash \eta(f)$ and we may isotope the decomposing tori, thereby redefining $M_{v}$ and the edge manifolds for which $e$ is incident to $v$ slightly, so that after this isotopy, $S \cap M_{v}$ is vertical and so that an edge manifold becomes the active component.

Case $2 S^{*} \cap\left(M_{v} \backslash \eta(f)\right)$ is vertical.

If $S^{*} \cap\left(M_{v} \backslash \eta(f)\right)$ is not boundary parallel, then it is essential and can't be contained in $V$ or $W$. This is a contradiction, hence this case does not occur. 
Case $3 S^{*} \cap\left(M_{v} \backslash \eta(f)\right)$ is horizontal.

Then $\left(S^{*} \cap\left(M_{v} \backslash \eta(f)\right)\right) \cup(S \cap N(f))$ is pseudohorizontal, but we must show that in fact $S \cap M_{v}=\left(S^{*} \cap\left(M_{v} \backslash \eta(f)\right)\right) \cup(S \cap N(f))$.

Since $\partial A$ has two components and since $S^{*} \cap\left(M_{v} \backslash \eta(f)\right)$ is separating there are two, necessarily parallel, components of $S^{*} \cap\left(M_{v} \backslash \eta(f)\right)$. Moreover, since $A$ is parallel into $\partial U$ in both directions, each component of $M_{v} \backslash S^{*}$ is homeomorphic to (punctured surface) $\times(0,1)$.

Suppose now that $S^{*}$ lies in, say, $V$. Denote the component of $M \backslash S^{*}$ that meets $W$ by $\hat{W}$. Then $S$ defines a Heegaard splitting of $\hat{W}$. Let $\mathcal{D}$ be a set of disks in the interior of $M_{v}$ that cut $\hat{W} \cap M_{v}$ into a collar of the annuli $\hat{W} \cap \partial M_{v}$. By Haken's Theorem, each such disk can be isotoped to intersect $S$ in a single circle. We may assume that after this isotopy, $\mathcal{D}$ still lies in the interior of $M_{v}$.

Here $S$ may be reconstructed by performing ambient 1 -surgery on $S^{*}$ along arcs in $\hat{W}$. But this collection of arcs is disjoint from $\mathcal{D}$. Thus all such arcs may be isotoped into edge manifolds $M_{e}$ such that $e$ is incident to $v$. Note that an edge manifold that contains such an arc becomes the active component. Also note that after this isotopy, the portion of $S$ remaining in $M_{v}$ is pseudohorizontal.

If a surface is pseudohorizontal, then it is boundary compressible. In particular, it lives in the active component. Hence the existence of such arcs contradicts Lemma 6.1 Thus $S \cap M_{v}=\left(S^{*} \cap\left(M_{v} \backslash \eta(f)\right)\right) \cup(S \cap N(f))$.

\subsection{Vertex manifold not Seifert fibered}

Next we consider the case in which the active component of $M=V \cup_{S} W$ is a vertex manifold $M_{v}$ that is homeomorphic to (compact orientable surface) $\times I$. The strategy here is an adaptation of the argument in the preceeding case. Though the setup here is much simpler.

Definition 7.11 Let $Q$ be a compact surface with non empty boundary. A spine of $Q$ is a 1 -complex $\Gamma^{1}$ with exactly one vertex $v$ that cuts $Q$ into a regular neighborhood of $\partial Q$. A 2-complex of the form $\Gamma^{2}=\Gamma^{1} \times I$ is called a spine of $Q \times I$. We denote the 1 -manifold $v \times I$ by $\gamma$.

The following lemma is another generalization of Lemma 3.11. We will only be interested in this lemma in the case that $N$ is a vertex manifold of a graph manifold, but again we state it in very general terms. It too applies to a larger class of 3-manifolds than just generalized graph manifolds. 
Lemma 7.12 Let $M=V \cup_{S} W$ be a strongly irreducible Heegaard splitting. Suppose $N$ is a submanifold of $M$ homeomorphic to (compact surface) $\times I$ such that (compact surface) $\times \partial I \subset \partial M$. Let $\Gamma^{2}$ be a spine of $N$. Then $S$ may be isotoped so that the following hold:

(1) $S \cap \Gamma^{2}$ consists of simple closed curves and simple closed curves wedged together at points in $\gamma$.

(2) No closed curve in $S \cap \Gamma^{2}$ bounds a disk in $\Gamma^{2} \backslash S$.

The proof of this lemma is identical to the proof of Lemma 7.7

Lemma 7.13 Suppose that the active component of $M=V \cup_{S} W$ is a vertex manifold $M_{v}$ that is homeomorphic to (compact orientable surface) $\times I$. Then we may isotope the decomposing annuli, thereby redefining the active component $M_{v}$ and the edge manifolds for which $e$ is incident to $v$ slightly, so that after this isotopy, $S \cap M_{v}$ is a horizontal incompressible surface and so that an edge manifold becomes the active component.

Proof Let $\Gamma^{2}$ be a spine of $M_{v}$ and isotope $S$ within $M_{v}$ so that the conclusions of Lemma 7.12 hold. Then $S \cap \Gamma^{2}$ consists of horizontal curves. Here $S \cap N\left(\Gamma^{2}\right)$ is a bicollar of $S \cap \Gamma^{2}$ and hence a horizontal incompressible surface.

Let $\tilde{M}_{v}$ be $N\left(\Gamma^{2}\right)$. Let $\tilde{A}$ be a component of $\partial \tilde{M}_{v} \backslash \partial M_{v}$. Then $\tilde{A}$ is parallel to a decomposing annulus $A$. We replace $A$ by $\tilde{A}$. We may do so via an isotopy. We do this for all components of $\partial \tilde{M}_{v} \backslash \partial M_{v}$. After this process, the conclusions of the lemma hold.

\subsection{Edge manifold homeomorphic to (annulus) $\times I$}

Now we consider the case in which the active component of $M=V \cup_{S} W$ is an edge manifold $M_{e}$ homeomorphic to (annulus) $\times I$. This case turns out to be a direct application of the following theorem of Marty Scharlemann:

Theorem 7.14 Suppose $M=V \cup_{S} W$ is a strongly irreducible Heegaard splitting and $U \subset M$ is a solid torus such that $S$ intersects $\partial U$ in parallel essential non meridional curves. Then $S$ intersects $U$ in a collection of boundary parallel annuli and possibly one other component, obtained from one or two annuli by ambient 1-surgery along an arc parallel to a subarc of $\partial U$. If the latter sort of component is in $U$, then $S \backslash U$ is incompressible in $M \backslash U$. 
Proof This is Theorem 3.3 in 17 .

The following proposition is stated in general terms. Our interest in this theorem will be the case in which $A \times I$ is an edge manifold. Since $A \times I$ is a solid torus, this proposition follows directly from Scharlemann's Theorem (Theorem 7.14).

Proposition 7.15 Suppose $A \times I$ is an imbedding of (annulus) $\times I$ in the interior of $M$ with $A \times\{$ point $\}$ essential in $M$. Further suppose that $M=$ $V \cup_{S} W$ is a strongly irreducible Heegaard splitting. If both components of $S \cap(A \times \partial I)$ consist of curves essential in both $S$ and $(A \times \partial I)$, then $S \cap$ $(A \times I)$ is isotopic to a collection of incompressible annuli and possibly one other component, obtained from two annuli by ambient 1-surgery along an arc parallel to a subarc of $A \times\{$ point $\}$.

The following definition clarifies the statement of Proposition 7.15 .

Definition 7.16 Let $A$ be an annulus and let $N=A \times I$. A spanning annulus for $N$ is an annulus of the form $c \times I$, for $c$ an essential curve in $A$. Similarly, let $T$ be a torus and $N=T \times I$. A spanning annulus for $N$ is an annulus of the form $c \times I$, for $c$ an essential curve in $T$.

Each of the incompressible annuli mentioned in Proposition 7.15 is either a spanning annulus or is parallel into $A \times \partial I$. In Figure 14 we see two isotopic possibilities. Note that the tube we see on the left hand side is actually "dual" to the tube we see on the right hand side.

The tube on the left hand side could (after straightening out the picture) be seen as ambient 1-surgery along an arc parallel to a subarc of $\{$ point $\} \times I$ (a vertical arc). In this case the the tube on the right hand side would be seen as ambient 1-surgery along an arc parallel to a subarc of $A \times\{$ point $\}$ (a horizontal arc).

\subsection{Edge manifold homeomorphic to $($ torus $) \times I$}

Next we consider the case in which the active component of $M=V \cup_{S} W$ is an edge manifold $M_{e}$ homeomorphic to (torus) $\times I$. The theorem proven here applies in very general contexts. The techniques used are those developed by Rubinstein and Scharlemann in [16]. 

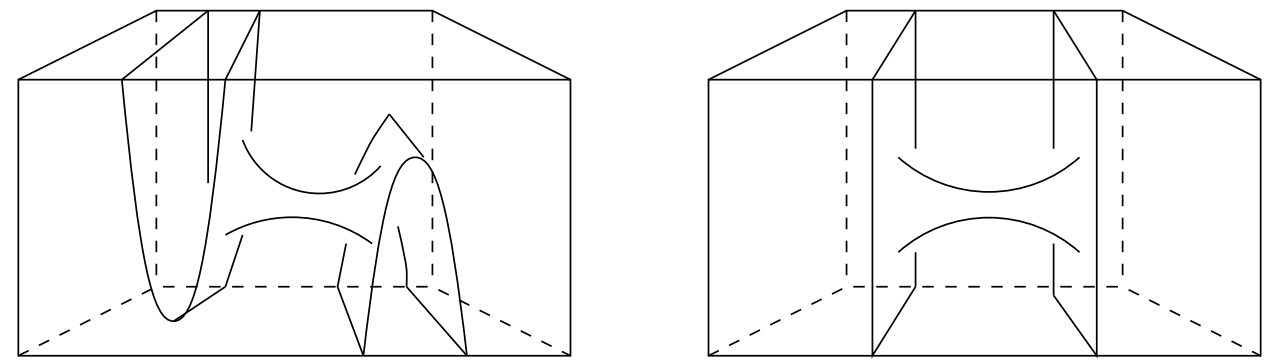

Figure 14: Two isotopic possibilities

The use of these techniques is inspired by Cooper and Scharlemann's application of the central argument in [16] to the setting of Heegaard splittings of solvmanifolds in [6, Theorem 4.2]. The arguments in this section are identical to the argument in [6. Theorem 4.2]. We weaken one of the hypotheses slightly. In addition, in the setting here, there are no constraints on $S \cap(T \times \partial I)$. In 6 a constraint arises due to the fact that in a solvmanifold the two components of $T \times \partial I$ are identified. This means that some scenarios that arise in the argument for [6. Theorem 4.2] can be ruled out there. But they can't be ruled out here. Thus our conclusions are slightly different.

The argument is rather lengthy. The reader is referred to [6] for a sketch and to 16 for details.

We recall some fundamentals concerning the Rubinstein-Scharlemann graphic. Let $\Sigma_{V}$ be a spine of $V$ and $\Sigma_{W}$ a spine of $W$. Then $M \backslash\left(\partial_{-} V \cup \Sigma_{V} \cup \partial_{-} W \cup \Sigma_{W}\right)$ is homeomorphic to $S \times I$. This product foliation is called a sweepout. If $S$ intersects a product submanifold $N=Q \times I$ of $M$ then to each point $(s, t)$ in $I \times I$ we may associate $S_{s}=S \times\{s\}$ and $Q_{t}=Q \times\{t\}$. We are interested in $S_{s} \cap Q_{t}$.

After a small isotopy, we may assume that the spines and the sweepout are in general position with respect to the foliation of $Q \times I$. There is then a $1-$ dimensional complex $\Gamma$ in $I \times I$ called the Rubinstein-Scharlemann graphic. See Figure 15. At a point $(s, t)$ away from $\Gamma, S_{s}$ and $Q_{t}$ are in general position. On an edge of $\Gamma$ the surfaces $S_{s}$ and $Q_{t}$ have a single point of tangency. At a vertex of $\Gamma$ there are either two points of tangency or a "birth-death" singularity.

For $(s, t)$ in a region of $(I \times I) \backslash \Gamma, S_{s} \cap Q_{t}$ is topologically rigid. If there is a curve $c$ in $S_{s} \cap Q_{t}$ that is essential in $S_{s}$ but bounds a disk in $Q_{t}$ that lies in $V$ near $c$, then we label the region $V$. Similarly, if there is a curve $c^{\prime}$ in $S_{s} \cap Q_{t}$ that is essential in $S_{s}$ but bounds a disk in $Q_{t}$ that lies in $W$ near $c$, then we label the region $W$. 


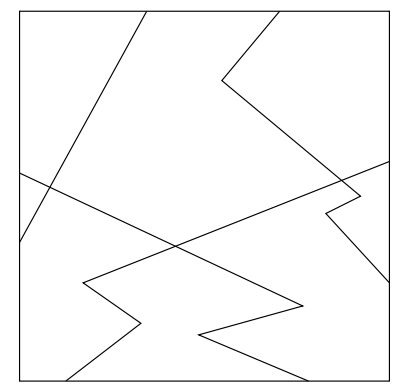

Figure 15: The Rubinstein-Scharlemann graphic

We summarize some of the insights from [16]:

Remark 7.17 If a region $R$ is labelled $V$, then for $(s, t) \in R$ the curve $c$ in $S_{s} \cap Q_{t}$ that is essential in $S_{s}$ and bounds a disk in $Q_{t}$ that lies in $V$ near $c$ also bounds a disk that lies entirely in $V$. The equivalent statement holds for the label $W$. (This is [16, Lemma 4.3].)

This fact has the following immediate consequences.

Remark 7.18 If a region is labelled both $V$ and $W$, then $M=V \cup_{S} W$ is weakly reducible. (This is [16, Corollary 4.4].)

Remark 7.19 If for some $s_{0} \in(0,1)$ and some $t_{0}, t_{1} \in(0,1)$ there is a region labelled $V$ containing $\left(s_{0}, t_{0}\right)$ and a region labelled $W$ containing $\left(s_{0}, t_{1}\right)$, then $M=V \cup_{S} W$ is weakly reducible.

Remark 7.19 is not explicitly stated in [16] but follows immediately from the definitions and Remark [7.17. Indeed, the labelling in the region containing $\left(s_{0}, t_{0}\right)$ gives an essential disk to one side of $S_{s_{0}}$ and the labelling in the region containing $\left(s_{0}, t_{1}\right)$ gives an essential disk to the other side of $S_{s_{0}}$. The boundaries of these disks are contained in $Q_{t_{0}}$ and $Q_{t_{1}}$ respectively. As $Q_{t_{0}}$ and $Q_{t_{1}}$ are disjoint, the boundaries of these disks are disjoint. Hence $M=V \cup_{S} W$ is weakly reducible.

More subtle facts are the following:

Remark 7.20 The labels $V$ and $W$ cannot both appear in regions adjacent along an edge. (This is [16, Corollary 5.5].) 
Notice that for small $s, S_{s} \cap(Q \times I)$ is the boundary of a small regular neighborhood of $\Sigma_{V} \cap(Q \times I)$. If the leaves of the foliation of $Q \times I$ are essential, then $\Sigma_{V}$ can't miss any such leaf. Similarly for $\Sigma_{W}$. Thus we have the following:

Remark 7.21 If the leaves of the foliation of $Q \times I$ are essential in $M$, then every region of $(I \times I) \backslash \Gamma$ abutting the left edge of $I \times I$ is labelled $V$. Every region of $(I \times I) \backslash \Gamma$ abutting the right edge of $I \times I$ is labelled $W$.

As a warm up in the application of these principles, we prove the following lemma:

Lemma 7.22 Suppose that $N=Q \times I$ is a product submanifold of $M$. Suppose further that $M=V \cup_{S} W$ is a strongly irreducible Heegaard splitting. Let $\Gamma$ be the Rubinstein-Scharlemann graphic and suppose there is a vertex $\left(s_{0}, t_{0}\right)$ of $\Gamma$ with the following properties:

(1) Four regions meet at $\left(s_{0}, t_{0}\right)$.

(2) One region is labelled $V$ and one region is labelled $W$.

Then the following hold:

(A) The other two regions abutting $\left(s_{0}, t_{0}\right)$ are unlabelled.

(B) The regions labelled $V$ and $W$ lie opposite each other.

(C) The graph $G=S_{s_{0}} \cap Q_{t_{0}}$ in $Q_{t_{0}}$ contains a connected subgraph $\tilde{G}$ with two vertices $v_{1}, v_{2}$, each of valence 4 .

(D) If an edge $e$ of $G$ has both ends on the same vertex $v$ then $e \cup v$ is an essential circle in $Q_{t_{0}}$.

(E) $\tilde{G}$ is not contractible in $Q_{t_{0}}$.

Proof Observations A and B follow directly from Remark [7.20, Recall that each edge signifies a tangency between $S_{s_{0}}$ and $Q_{t_{0}}$. If the tangency corresponds to a maximum or minimum, then the labelling of adjacent regions does not change as the edge is traversed. So here the tangencies corresponding to edges must arise from saddle singularities.

It follows that $S_{s_{0}} \cap Q_{t_{0}}$ consists of a graph $G$ in $Q_{t_{0}}$ that has two valence four vertices. In the four regions abutting $\left(s_{0}, t_{0}\right)$ the valence four vertices break apart in four possible combinations. See Figure 16

Suppose the two valence four vertices of $G$ lie on distinct components $\tilde{G}_{1}, \tilde{G}_{2}$ of $G$. As $\tilde{G}_{1}, \tilde{G}_{2}$ break apart as in Figure 16] the curves arising from $\tilde{G}_{1}$ remain 


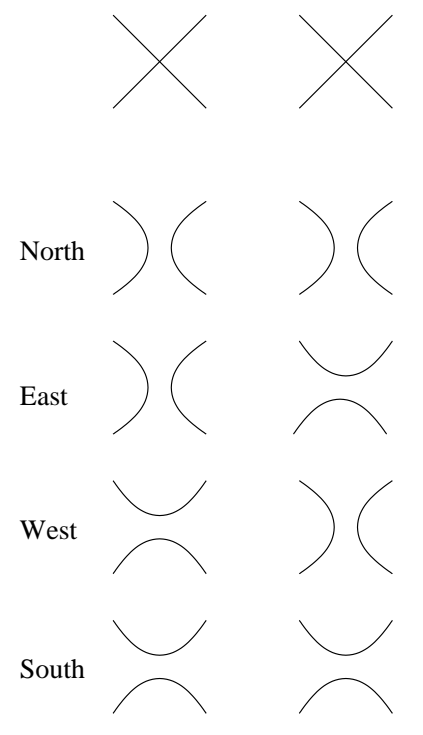

Figure 16: How $S_{s}$ meets $T_{t}$ in the four regions North, East, West and South meeting $\left(s_{0}, t_{0}\right)$

disjoint from the curves arising from $\tilde{G}_{2}$. Furthermore, the curves arising from $\tilde{G}_{1}$ are identical in the regions North and East and in the regions West and South. The curves arising from $\tilde{G}_{2}$ are identical in the regions North and West and in the regions East and South. It follows that one of the components, say $\tilde{G}_{1}$, must give rise to the labelling $V$ and the other component, say $\tilde{G}_{2}$, must give rise to the labelling $W$. But then two adjacent regions, say the regions North and East, are labelled $V$. A contradiction. Thus $\mathrm{C}$ holds.

Denote the vertices by $v_{1}$ and $v_{2}$. Suppose that $e$ has both ends on $v_{1}$ and that $v_{1} \cup e$ is inessential. Then there will be two adjacent regions, say the regions North and East, in which this monogon gives rise to a simple closed curve. Since one of the regions, say North, is labelled, say $V$, this simple closed curve must give rise to a labelling $V$. But then it gives rise to this labelling in both the region North and in the region East. A contradiction. Hence D holds.

Finally, suppose $\tilde{G}$ is contractible in $Q_{t_{0}}$. By D, each edge has endpoints on distinct vertices. Thus $\tilde{G}$ is as in Figure [17. Hence in the regions North, East, West and South of $(I \times I) \backslash \Gamma$ we see components of intersection of $S_{s} \cap Q_{t}$ in $Q_{t}$ as in Figure 18.

The curves of intersection pictured are the ones giving rise to the labellings. Note that if the circles pictured in East and West give rise to labellings, then 


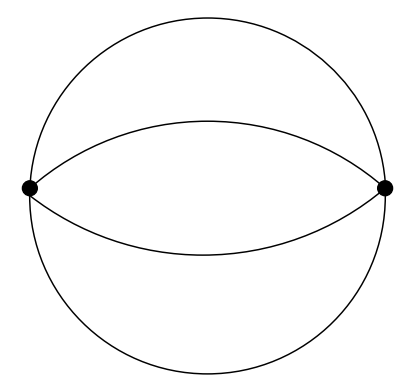

Figure 17: $\tilde{G}$ is contractible
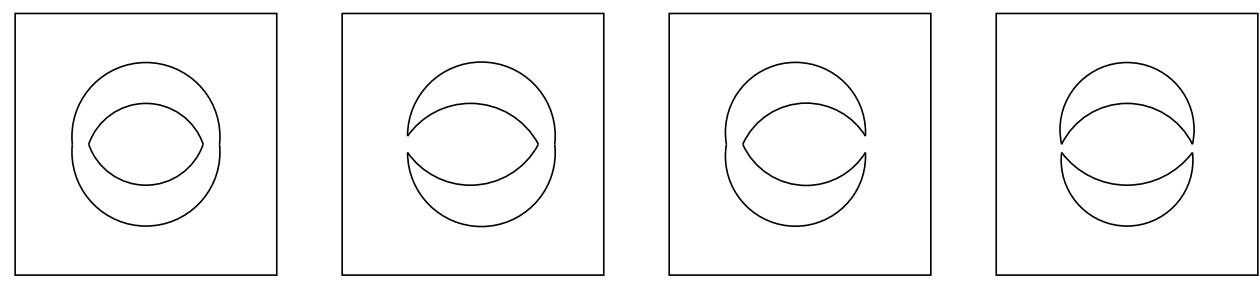

Figure 18: The regions North, East, West and South

they give rise to the same labelling. Hence these regions must both be unlabelled. It follows that North and South are the labelled regions. If the larger circle pictured in North gives rise to a labelling, then this labelling coincides with the labelling arising from the circle(s) pictured in South. But this is impossible. Thus the smaller circle pictured in North gives rise to the labelling. We may assume that it gives rise to the label $V$.

Let $(s, t) \in$ North. Denote the annulus cut out by the two circles in North by $\tilde{A}$. Denote the disk cut out by the inner circle by $\tilde{D}$. By Remark 7.17 we may assume that $\tilde{D} \subset V$. Denote the outer component of $\partial \tilde{A}$ by $c$ and the inner component by $c^{\prime}$. Since $c^{\prime}$ gives rise to a labelling, $c^{\prime}$ is essential in $S_{s}$. Since $c$ does not give rise to a labelling, $c$ is inessential in $S_{s}$. Let $D \subset S_{s}$ be the disk bounded by $c$. We may assume that this disk is disjoint from $c \cup c^{\prime}$. After a small isotopy, $\tilde{A} \cup D$ is an essential disk in $W$. But here $\partial(\tilde{A} \cup D)=\partial \tilde{D}$. So $M=V \cup_{S} W$ is reducible. But this is impossible. Thus $\tilde{G}$ is not contractible in $Q_{t_{0}}$. Hence E holds.

The following proposition gives the required information concerning the intersection of a strongly irreducible Heegaard splitting with an edge manifold homeomorphic to (torus) $\times I$ when this edge manifold is the active component. 
Proposition 7.23 Suppose $T \times I$ is an imbedding of (torus) $\times I$ in the interior of $M$ with $T \times\{t\}$ essential in $M$. Further suppose that $M=V \cup_{S} W$ is a strongly irreducible Heegaard splitting. If $S \cap(T \times \partial I)$ consist of curves essential in both $S$ and $(T \times \partial I)$, then $S \cap(T \times I)$ is characterized by one of the following:

(1) $S \cap(T \times I)$ is isotopic to a collection of incompressible annuli and possibly one other component, obtained from two such annuli by ambient 1-surgery along an arc parallel to a subarc of $T \times\{$ point $\}$.

(2) There is a pair of simple closed curves $c, c^{\prime} \subset T$ such that $c \cap c^{\prime}$ consists of a single point $p \in T$ and $V \cap(T \times I)$ is a collar of $(c \times\{0\}) \cup(p \times I) \cup\left(c^{\prime} \times\{1\}\right)$.

Proof Consider the regions of $(I \times I) \backslash \Gamma$ abutting $I \times\{0\}$. Since $S \cap(T \times \partial I)$ consist of curves essential in both $S$ and $(T \times \partial I)$ there must be an unlabelled such region. We call this region $R_{0}$. Similarly, there must be an unlabelled region, which we call $R_{1}$, that abuts $I \times\{1\}$. Because of the conditions on $S \cap(T \times \partial I)$ we may assume that for $(s, t)$ in $R_{0}$ or $R_{1}, S_{s} \cap T_{t}$ consists of curves essential in both $S_{s}$ and $T_{t}$. We are interested in monotone paths from $R_{0}$ to $R_{1}$.

Our argument breaks into two cases: Either there is a path in $I \times I$ from $R_{0}$ to $R_{1}$ that avoids labelled regions or there is no such path.

Case 1 There is a path beginning in $R_{0}$ and ending in $R_{1}$ that traverses only unlabelled regions and edges between such regions. See Figure 19.

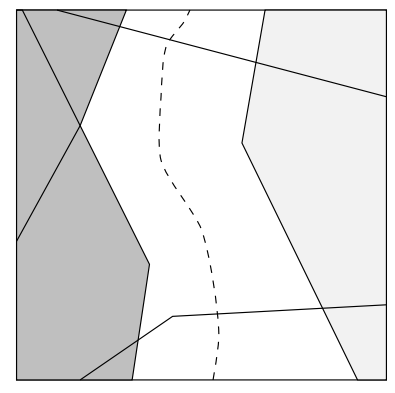

Figure 19: A monotone path through unlabelled regions

It follows from Remark 7.19 that there is a monotone path $\alpha$ beginning in $R_{0}$ and ending in $R_{1}$ that traverses only unlabelled regions and edges between such regions.

Consider the effect of traversing an edge $e$ from one unlabelled region of $(I \times$ $I) \backslash \Gamma$ to another. Since compression bodies do not contain essential surfaces, 
$S_{s} \cap T_{t}$ must contain curves essential in $S_{s}$. For $(s, t)$ in an unlabelled region, such curves must also be essential in $T_{t}$. We may thus refer to curves inessential in both $S_{s}$ and $T_{t}$ simply as inessential and to curves essential in both $S_{s}$ and $T_{t}$ simply as essential.

As $\alpha$ crosses $e$, the components of intersection of $S_{s} \cap T_{t}$ change in the same way that level curves change, as we rise from being below to being above a maximum, minimum or saddle point. If the point of tangency corresponding to $e$ corresponds to a maximum or minimum, then an inessential curve appears or disappears. If the point of tangency corresponds to a saddle singularity, then either one curve is banded to itself or two curves are banded together. Note that $S_{s} \cap T_{t}$ is separating in $T_{t}$, hence the essential curves come in pairs parallel in $T_{t}$. Thus if a curve is banded to itself, then any resulting essential curves are parallel, in $T_{t}$, to other essential curves. If two essential curves are banded to each other, then an inessential curve results, but essential curves must remain. In both cases, the slope, in $T_{t}$, of the essential curves is unaffected, as $t$ increases.

Since $\alpha$ is monotone, we obtain a function $f: I \rightarrow I$ by requiring $f(t)$ to be the value $s$ such that $(s, t)$ is in the image of $\alpha$. We may now isotope $S \cap(T \times I)$ by isotoping $S \cap(T \times\{t\})$ to $S_{f(t)} \cap T_{t}$ for each $t \in I$. Thus $\alpha$ describes an isotopy of $S$. We will assume in the following that this isotopy has been performed. The preceding paragraph tells us how $S \cap(T \times\{t\})$ changes as $t$ increases. We must reconstruct $S \cap(T \times I)$ from these level sets.

As $\alpha$ traverses an unlabelled region, the curves of intersection $S \cap T_{t}$ sweep out annuli. As $\alpha$ crosses an edge corresponding to a tangency corresponding to a maximum or minimum an inessential curve appears or disappears. If such a curve appears, this indicates the appearance of a disk. If such a curve disappears, this indicates that an inessential curve is capped off by a disk. Note that since there are no disks for $t=0$ or for $t=1$, these disks merely cap off inessential curves. Converserly, inessential curves are always capped off by such disks.

Suppose that $\alpha$ crosses an edge corresponding to a tangency corresponding to a saddle point at $\left(s_{0}, t_{0}\right)$. If an inessential curve is banded to itself, then there are two possibilities:

(1) Two inessential curves result. These curves are nested in $T_{t_{0}+\epsilon}$. However, one of these inessential curves lies in the subdisk of $S$ bounded by the other. Hence the appearance of the new inessential curve does not indicate the appearance of another disk. The appearance of the new inessential curve 
merely indicates a saddle singularity, with respect to projection onto $t$, in the imbedding of a single disk. Thus we may ignore this phenomenon.

(2) Two essential curves result. Since the inessential curve bounds a disk in $S$, the components of $S \cap T_{t}$ affected piece together to form an annulus that is parallel into $T_{t_{0}+\epsilon}$.

If an essential curve is banded to itself, then either one essential curve or one inessential and one essential curve result. But the first possibility can't occur here, because here $S \cap T_{t}$ is separating for all $t$. Thus one inessential and one essential curve result. The inessential curve bounds a disk in $S$. Hence the appearance of the new inessential curve merely indicates a saddle singularity, with respect to projection onto $t$, in the imbedding of an essential annulus. Thus we may again ignore this phenomenon.

Finally, suppose that a pair of essential curves disappears as two essential curves are banded to each other to produce an inessential curve. The inessential curve bounds a disk in $S$. Thus the components of $S \cap T_{t}$ affected piece together to form an annulus that is parallel into $T_{t_{0}-\epsilon}$.

Since there are no inessential curves for $t=0$ or $t=1$, we see that, after further isotopies, $S \cap(T \times I)$ consists of spanning annuli and annuli parallel into $T \times \partial I$.

Case 2 There is no path beginning in $R_{0}$ and ending in $R_{1}$ that traverses only unlabelled regions and edges between such regions. See Figure 20.

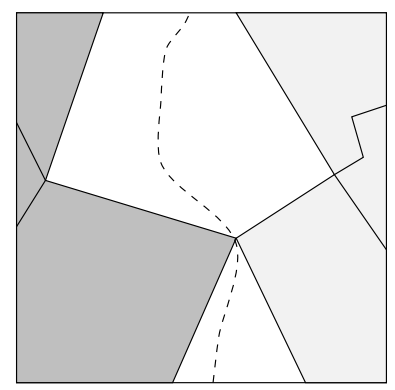

Figure 20: A monotone path through unlabelled regions

In this case the regions of $(I \times I) \backslash \Gamma$ labelled $V$ extending from the left edge of $I \times I$ must meet the regions of $(I \times I) \backslash \Gamma$ labelled $W$ extending from the right edge of $I \times I$. By Remark 7.20 this can only happen at a vertex. Thus Lemma 7.22 applies. 
We consider $\tilde{G}$ as in C) of Lemma 7.22 Denote the vertices by $v_{1}, v_{2}$ and the edges by $e_{1}, e_{2}, e_{3}, e_{4}$. Suppose first that one edge, say $e_{1}$ has both ends on $v_{1}$. Then since $\tilde{G}$ is connected, and since $v_{1}$ and $v_{2}$ have valence four, there must be exactly two edges $e_{2}, e_{3}$, each with one end on $v_{1}$ and one end on $v_{2}$. It follows that $e_{4}$ must have both ends on $v_{2}$. By Lemma 7.22 both $v_{1} \cup e_{1}$ and $v_{2} \cup e_{4}$ are essential. Since these curves lie on a torus, they are parallel. The other possibility is that all edges have one end on $v_{1}$ and one end on $v_{2}$.

Subcase 2.1 $e_{1}$ has both ends on $v_{1}, e_{4}$ has both ends on $v_{2}$ and $e_{2}$ and $e_{3}$ are parallel.

Here $\tilde{G}$ is as in Figure 21.

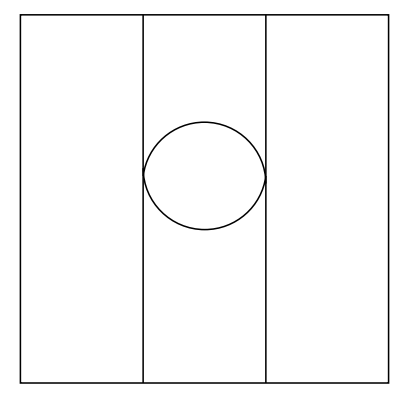

Figure 21: $\tilde{G}$ contains a bigon

This implies that as $v_{0}$ is crossed going from one unlabelled region to another, $S \cap T_{t}$ changes as in Figure 22
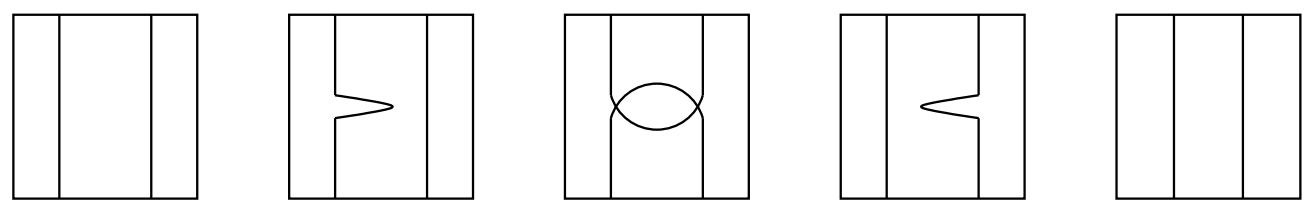

Figure 22: Adding a 1-handle

But this corresponds to the addition of a 1-handle. The core of the 1-handle can be isotoped into $T_{t_{0}}$. Since there are essential disks for both $V$ and $W$ cut out by $\tilde{G}, S \backslash\left(T \times\left[t_{0}-\epsilon, t_{0}+\epsilon\right]\right)$ is incompressible in $M \backslash\left(T \times\left[t_{0}-\epsilon, t_{0}+\epsilon\right]\right)$. In particular, we may assume that $S \cap\left(T \times\left\{t_{0}-\epsilon, t_{0}+\epsilon\right\}\right)$ consists of curves essential in $S$ and in $T_{t_{0} \pm \epsilon}$. This implies that $S \cap\left(T \times\left[t_{0}-\epsilon, t_{0}+\epsilon\right]\right)$ is as described in option (1).

To see that option (1) holds for all of $S \cap(T \times I)$, note that $S \cap((T \times I) \backslash(T \times$ $\left.\left.\left[t_{0}-\epsilon, t_{0}+\epsilon\right]\right)\right)$ consists of incompressible annuli. Spanning annuli merely extend 
components of $S \cap\left(T \times\left[t_{0}-\epsilon, t_{0}+\epsilon\right]\right)$. Annuli parallel into $T_{t_{0} \pm \epsilon}$ create annuli parallel into $T \times \partial I$ unless they meet the compressible component of $S \cap\left(T \times\left[t_{0}-\epsilon, t_{0}+\epsilon\right]\right)$.

The question is thus merely whether the ends of the compressible component of $S \cap\left(T \times\left[t_{0}-\epsilon, t_{0}+\epsilon\right]\right)$ meet spanning annuli or annuli parallel into $T_{t_{0} \pm \epsilon}$. There are a number of possibilities. But all possibilities are either as described in option (1) or lead to a contradiction, by implying that $S$ is stabilized. Some options are pictured in Figure 23. Figure 24, Figure 25.
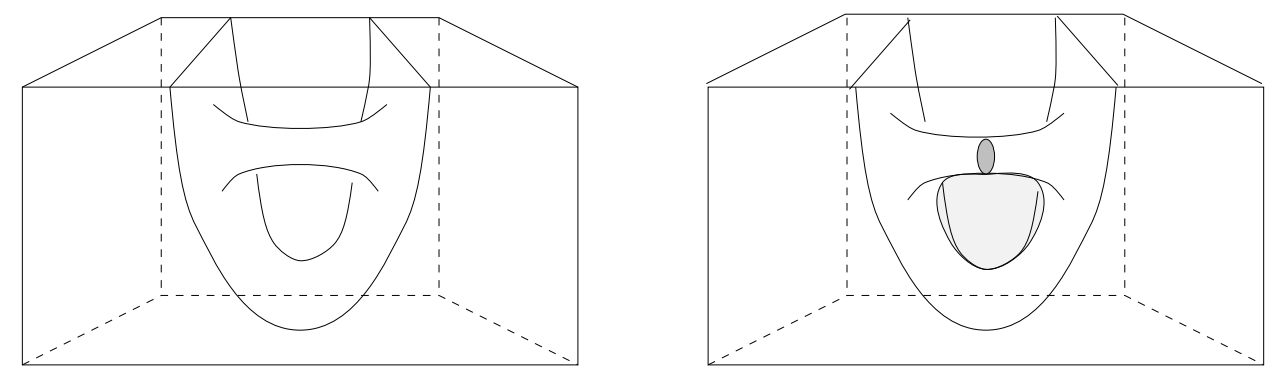

Figure 23: Compressible component meets annulus parallel into $T_{t_{0} \pm \epsilon}$
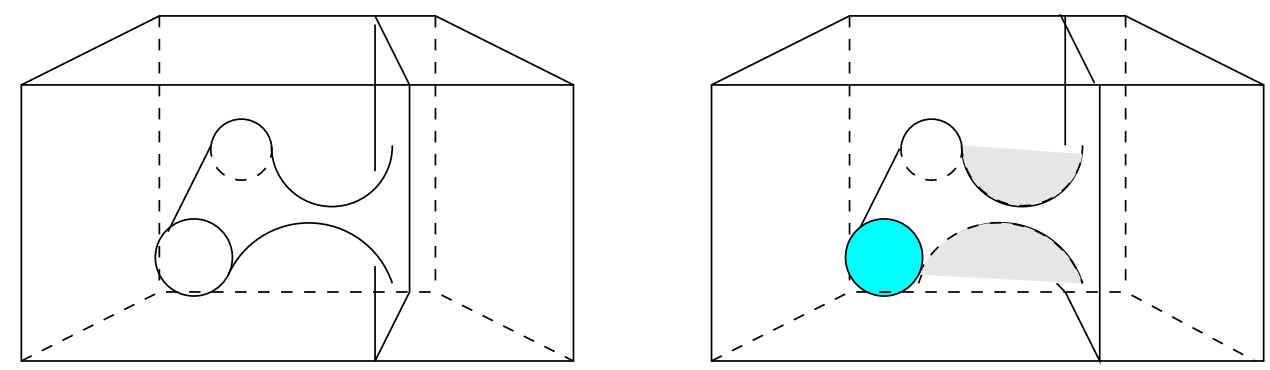

Figure 24: Another stabilized possibility

Subcase 2.2 $e_{1}$ has both ends on $v_{1}, e_{4}$ has both ends on $v_{2}$ and $e_{2}$ and $e_{3}$ are not parallel.

Here $\tilde{G}$ is as in Figure 26.

This implies that as $v_{0}$ is crossed going from one unlabelled region to another, $S \cap T_{t}$ changes as in Figure 27

Thus $S \cap T \times\left[t_{0}-\epsilon, t_{0}+\epsilon\right]$ consists of spanning annuli together with one component that is obtained as follows: an annulus that is parallel into $T_{t_{0} \pm \epsilon}$ is tubed to a spanning annulus. This is precisely the type of compressible 

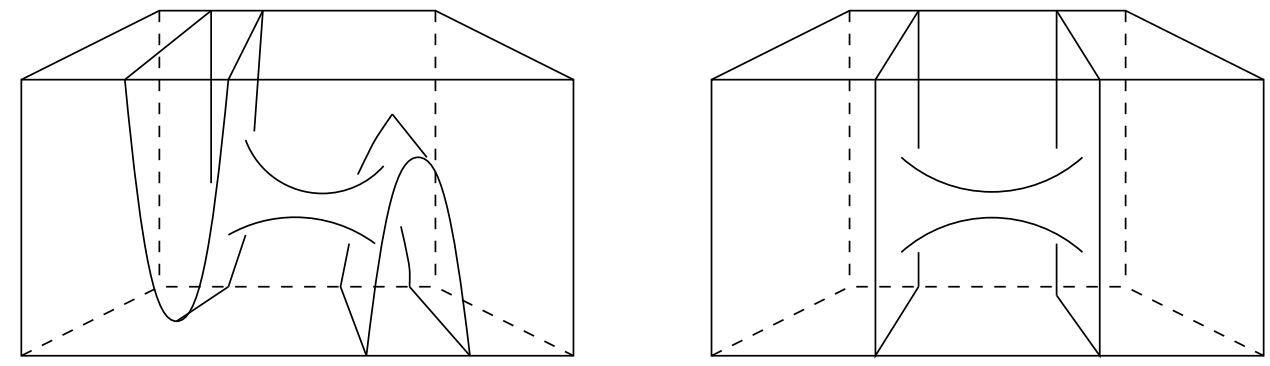

Figure 25: Two isotopic possibilities

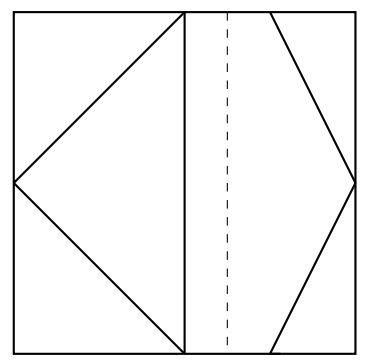

Figure 26: $\tilde{G}$ contains a triangle (dashed arc included along with $\tilde{G}$ to indicate that a bicoloring, as required, is possible)
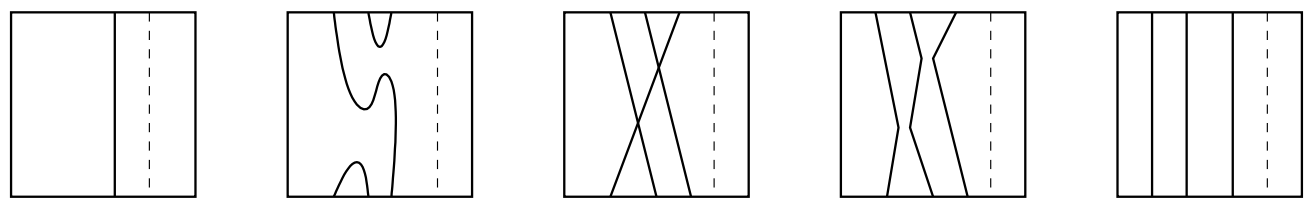

Figure 27: Adding an "essential" 1-handle

component that arises in Case 2.1 if an annulus parallel into $T_{t_{0}-\epsilon}$ is attached to one end of the compressible component and one end of a spanning annulus. Thus as in Subcase 2.1, all unstabilized configurations involving $S \cap T \times\left[t_{0}-\right.$ $\left.\epsilon, t_{0}+\epsilon\right]$ are as described in option (1).

Subcase 2.3 All edges have one end on $v_{1}$ and the other on $v_{2}$.

Note that $S$ is separating. This induces a bicoloring of $T_{t_{0}} \backslash S$. Such a bicoloring is not possible if two edges are parallel. This forces $\tilde{G}$ to be as in Figure 28,

This implies that as $v_{0}$ is crossed going from one unlabelled region to another, $S \cap T_{t}$ changes as in Figure 29.

In particular, $S \cap T \times\left[t_{0}-\epsilon, t_{0}+\epsilon\right]$ is as in option (2). Again, $S \cap((T \times I) \backslash(T \times$ 


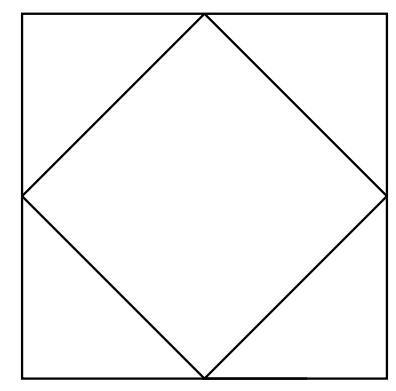

Figure 28: $\tilde{G}$ contains a square
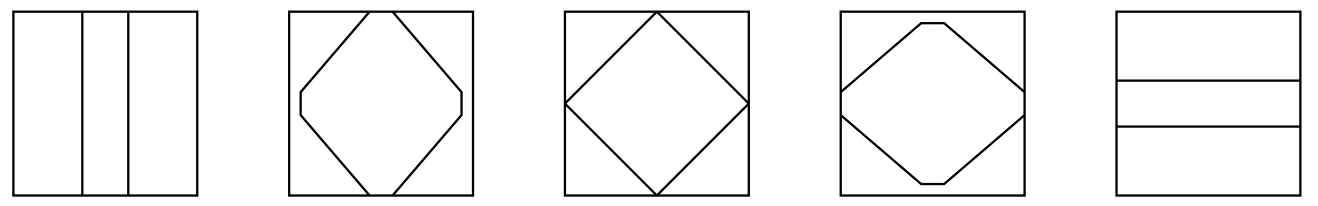

Figure 29: Another way of adding a 1-handle

$\left.\left.\left[t_{0}-\epsilon, t_{0}+\epsilon\right]\right)\right)$ consists of incompressible annuli. If an end of $S \cap T \times\left[t_{0}-\epsilon, t_{0}+\epsilon\right]$ meets an annulus that is not isotopic to a spanning annulus, then both ends meet this annulus. But this would imply that $S \cap(T \times I)$ is disconnected. Hence the ends of $S \cap T \times\left[t_{0}-\epsilon, t_{0}+\epsilon\right]$ meet spanning annuli and $S \cap(T \times I)$ is as in option (2). See Figure 29

\section{Fitting the pieces together}

We here prove the main theorem. As it turns out, the hard work is already done. It remains to fit the results together.

Recall that graph manifolds with empty characteristic submanifolds are Seifert fibered spaces. The corresponding result for Seifert fibered spaces was obtained by Y. Moriah and the author in [13] together with 21] and 22. Generalized graph manifolds with empty characteristic submanifolds are either Seifert fibered spaces or product manifolds of the form $($ surface $) \times I$. The corresponding result for such manifolds was obtained by M. Scharlemann and A. Thomspon in [19].

Proof of Theorem 1.1 If $\mathcal{E}=\emptyset$, then $S$ is either pseudohorizontal or pseudovertical, by the main theorems of [19, [13, 21] and [22. Thus we may assume in what follows that $\mathcal{E} \neq \emptyset$. 
Let $\mathcal{T}$ be the collection of decomposing annuli and tori. Let $N$ be a component of $M \backslash \mathcal{T}$. By Lemma 6.1 $S \cap N$ is incompressible unless $N$ is the active component. Thus if $N$ is not the active component, then $S \cap N$ is as required. Specifically, if $N$ is a vertex manifold, $S \cap N$ is either horizontal or vertical. And if $N$ is an edge manifold, then $S \cap N$ consists of incompressible annuli.

If $N$ is the active component, we must consider the possibilities: If $N$ is a vertex manifold that is Seifert fibered and has non empty external boundary then Proposition 7.5 applies. If $N$ is a vertex manifold that is a product, then Lemma 7.13 applies. Thus in these two cases, we may rechoose the decomposing annuli or tori so that an edge manifold becomes the active component. Similarly, if $N$ is vertex manifold that is a Seifert fibered space with no external boundary, then either $S \cap N$ is pseudohorizontal, or we may rechoose the decomposing tori so that an edge manifold becomes the active component.

Finally, if $N$ is an edge manifold, then $S \cap N$ is as required by Proposition 7.15 and Proposition 7.23 .

Now Theorem 1.2 and Theorem 1.3 follow from Theorem 1.1 along with the constructions of untelescoping and amalgamation. Recall Theorem 3.10 which states that, given an irreducible Heegaard splitting, the amalgamation of the weak reduction of this Heegaard splitting yields the original Heegaard splitting.

Note that the amalgamation of strongly irreducible Heegaard splittings of generalized graph manifolds interferes with the structure of the Heegaard splittings on the generalized graph manifolds that are being amalgamated. For this reason, we can't prove 1.1 without the hypothesis of strong irreducibility. Recall Example 5.6. which illustrates the obstruction.

Proof of Theorem 1.2 Consider the irreducible Heegaard splitting $M=$ $V \cup_{S} W$. Let $M=\left(V_{1} \cup_{S_{1}} W_{1}\right) \cup_{F_{1}} \cdots \cup_{F_{n-1}}\left(V_{n} \cup_{S_{n}} W_{n}\right)$ be a weak reduction of $M=V \cup_{S} W$. Denote the decomposing tori by $\mathcal{T}$. By Lemma 4.2, $\cup_{i} F_{i}$ can be isotoped so that for each vertex manifold $M_{v}$ of $M, F_{i} \cap M_{v}$ is either horizontal or vertical and so that for each edge manifold $M_{e}, F_{i} \cap M_{e}$ consists of incompressible tori and essential annuli. Hence, cutting $M$ along $\cup_{i} F_{i}$ consists of generalized totally orientable graph manifolds.

Since $M=\left(V_{1} \cup_{S_{1}} W_{1}\right) \cup_{F_{1}} \cdots \cup_{F_{n-1}}\left(V_{n} \cup_{S_{n}} W_{n}\right)$ is a weak reduction of $M=V \cup_{S} W$, each $M_{i}=V_{i} \cup_{S_{i}} W_{i}$ is strongly irreducible. Thus $M_{i}=V_{i} \cup_{S_{i}} W_{i}$ is a strongly irreducible Heegaard splitting of a generalized graph manifold and Theorem 1.1 applies. The result now follows by juxtaposing the strongly irreducible Heegaard splittings of the generalized graph manifolds. 
The Euler characteristic calculation follows directly from the definition of a weak reduction of a Heegaard splitting along with the fact that here $\chi(\partial M)=0$.

Proof of Theorem 1.3 This follows immediately from Theorems 3.10 and 1.2

Note that a horizontal (or a pseudohorizontal, respectively) surface in a vertex manifold corresponds to a foliation of that manifold as a surface bundle over the circle (or to a foliation of that manifold minus a vertical solid torus as a surface bundle over the circle). If the splitting surface of a Heegaard splitting can be isotoped so as to be horizontal or pseudohorizontal in two adjacent vertex manifolds, then the glueing data must satisfy certain restrictions. It is thus often the case that the splitting surface of a strongly irreducible Heegaard splitting of a graph manifold can't be isotoped to be horizontal or pseudohorizontal in adjacent vertex manifolds. Consequently, the canonical example of a Heegaard splitting of a graph manifold would seem to be a Heegaard splitting obtained by taking Heegaard splittings of the vertex manifolds with pseudovertical splitting surfaces and amalgamating these to obtain a Heegaard splitting of the graph manifold.

\section{References}

[1] M Boileau, J-P Otal, Groupes des difféotopies de certaines variétés de Seifert, C.R. Acad. Sci. Paris 303-I (1986) 19-22, MR87g:57022

[2] M Boileau, J-P Otal, Scindements de Heegaard et groupes des homotopies des petites varietes de Seifert, Invent. Math. 106 (1991) 85-107

[3] F Bonahon, J-P Otal, Scindements de Heegaard des espaces lenticulaires, Ann. Scient. Ec. Norm. Sup. 16 (1983) 451-466

[4] G Burde, H Zieschang, Knots, de Gruyter Studies in Mathematics, 5. Walter de Gruyter \& Co. Berlin (1985)

[5] A Casson, C Gordon, Reducing Heegaard splittings, Topology and its Applications 27 (1987) 275-283

[6] D Cooper, M Scharlemann, The structure of a solvmanifold's Heegaard splittings from: "Proceedings of 6th Gökova Geometry-Topology Conference", Turkish J. Math. 23 (1999) 1-18, arXiv:math.GT/9803157

[7] D B A Epstein, Periodic flows on 3-manifolds Ann. Math. 95 (1972) 68-82

[8] D Gabai, Foliations and the topology of 3-manifolds III, J. Differential Geom. 26 (1987) 479-536 
[9] J Hempel, 3-manifolds, Annals of Mathematics Studies 86, Princeton University Press, Princeton, NJ (1976)

[10] W Jaco, Lectures on Three-Manifold Topology, CBMS Lecture Notes, No. 43, Amer. Math. Soc. Providence, RI (1980) MR81k:57009

[11] W B Raymond Lickorish, An introduction to knot theory, Graduate Texts in Mathematics 175, Springer-Verlag, New York (1997)

[12] J Milnor, Lectures on the $h$-cobordism theorem, notes by L Siebenmann and L Sondow, Princeton Mathematical Notes, Princeton University Press (1965)

[13] Y Moriah, J Schultens, Irreducible Heegaard splittings of Seifert fibered spaces are horizontal or vertical, Topology 37 (1998) 1089-1112

[14] J. Nielsen, Abbildungsklassen endlicher Ordnung, Acta Mathematica 75 (1943) $23-115$

[15] Dale Rolfsen, Knots and links, Corrected reprint of the 1976 original, Mathematics Lecture Series, 7, Publish or Perish, Inc. Houston, TX (1990)

[16] J Hyam Rubinstein, M Scharlemann, Comparing Heegaard splittings of non-Haken 3-manifolds Topology 35 (1996) 1005-1026

[17] M Scharlemann, Local detection of strongly irreducible Heegaard splittings Topology and its Applications 90 (1998) 135-147

[18] M Scharlemann, A Thompson, Thin position for 3-manifolds AMS Contemporary Math. 164 (1994) 231-238

[19] M Scharlemann, A Thompson, Heegaard splittings of (surface) $\times I$ are standard, Math. Ann. 295 (1993) 549-564

[20] J Schultens, The Classification of Heegaard splittings for (closed orientable surface) $\times S^{1}$, London Math. Soc. 67 (1993) 425-448

[21] J Schultens, Heegaard splittings of Seifert fibered spaces with boundary, Trans. Amer. Math. Soc. 347 (1995) 2533-2552

[22] J Schultens, Weakly reducible Heegaard splittings of Seifert manifolds are vertical, Topology Appl. 100 (2000) 219-222

[23] J Schultens, Additivity of Tunnel Number for Small Knots, Comment. Math. Helv. 75 (2000) 353-367

[24] J Schultens, R Weidmann, On the geometric and algebraic rank of graph manifolds, preprint

[25] William P Thurston, Silvio Levy, editor, Three-dimensional geometry and topology. Vol. 1 Princeton Mathematical Series, 35. Princeton University Press, Princeton, NJ (1997) ISBN: 0-691-08304-5

[26] R Weidmann, Some 3-manifolds with 2-generated fundamental group, to appear in Arch. Math. 